\title{
Geometric Analysis of an Integrated Pest Management Model Including Two State Impulses
}

\author{
Wencai Zhao, Yulin Liu, Tongqian Zhang, and Xinzhu Meng \\ School of Mathematics and Systems Science, Shandong University of Science and Technology, Qingdao 266590, China \\ Correspondence should be addressed to Wencai Zhao; zhaowencai@sdust.edu.cn
}

Received 10 December 2013; Accepted 20 January 2014; Published 19 March 2014

Academic Editor: Kaifa Wang

Copyright ( 2014 Wencai Zhao et al. This is an open access article distributed under the Creative Commons Attribution License, which permits unrestricted use, distribution, and reproduction in any medium, provided the original work is properly cited.

\begin{abstract}
According to integrated pest management strategies, we construct and investigate the dynamics of a Holling-Tanner predator-prey system with state dependent impulsive effects by releasing natural enemies and spraying pesticide at different thresholds. Applying the Dulacs criterion, the global stability of the positive equilibrium in the system without impulsive effect is discussed. By using impulsive differential equation geometry theory and the method of successor functions, we prove the existence of periodic solution of the system with state dependent impulsive effects. Furthermore, the stability conditions of periodic solutions are obtained. Some simulations are exerted to illustrate the feasibility of our main results.
\end{abstract}

\section{Introduction}

In 2012, the main maize area in Northern China embraced the large-scale plant diseases and insect pests [1]. With the good weather condition, Mythimna separata Walker, Ostrinia furnacalis, aphid, and other pests had a mass propagation which brought a large damage to the production of corn. How human beings effectively control the pests has been a significant task. Due to its simple operation and quick effect, spraying pesticide has always been the major way to kill pests for a long time. However, there may be pesticide residues in vegetables and crops threatening people's good health and damaging the environment. With the increasing awareness of the environment, people are paying more attention to developing green agriculture. Releasing natural enemies and artificial capture are becoming significant means of controlling pests.

Different control strategies will be applied due to different behavior features of pests and their damages for crops in different stages. For instance, in the spawning period of Ostrinia furnacalis, artificial releasing of Trichogramma (the natural enemy of Ostrinia furnacalis) will be applied with the control effect of $70 \%-80 \%$; if the hatchability is over $30 \%$, pesticide control will be used instantly [2]. In consideration of the rapidity of chemical control and nonpollution of biological control, people control pests integrating with biological, physical, and chemical means under the EIL (economic injury level) to realize environmental, economic, and social profits together. Spraying pesticides and releasing natural enemies are instantaneous; these phenomena can be described as the impulsive differential equations. In recent decades, the theoretical research on the impulsive differential equation has represented a significant development and has been widely used in various mathematical ecological models [3-11] and many scholars made a deep analysis of the impulsive differential ecological system at a fixed time and have got some important products [12-26]. However, in the actual process of pest control, relevant measures will be used according to pest quantity and its damage to crops, which is the state impulsive differential system. Tang et al. [27, 28], Zeng et al. [29], Zhao et al. [30], Nie et al. [31, 32], and literatures [33-37] had a further exploration for the system and made great progress. Based on the study of a HollingTanner system, an integrated pest control model with two impulses is established aiming at the specific pest conditions in different threshold.

Let $x=x(t), y=y(t)$ denote the population densities of pest (prey) and natural enemy (predator) at time $t$, 
respectively; then the predator-prey system usually can be expressed as [38]

$$
\begin{gathered}
\frac{d x}{d t}=x g(x)-y \Phi(x), \\
\frac{d y}{d t}=y[-q(x)+c \Phi(x)],
\end{gathered}
$$

in which $g(x)$ denotes the relative growth rate of the prey; $\Phi(x)$ is the functional response function of the predator; $q(x)$ is the mortality rate of the prey. The literature [39] studied a class of Holling-Tanner system with functional response function $\Phi(x)=m x /(A+x)$. This system was given by

$$
\begin{gathered}
\frac{d x}{d t}=r x\left(1-\frac{x}{K}\right)-\frac{m x}{A+x} y, \\
\frac{d y}{d t}=y s\left(1-\frac{h y}{x}\right), \\
x(0)>0, y(0)>0 .
\end{gathered}
$$

Here, let the prey population be growth in logistic and the environmental capacity is $K$; the intrinsic growth rate of predator is $s$ and the carrying capacity is proportional to the number of prey. Introducing transformation $\widetilde{t}=r t, \widetilde{x}(\widetilde{t})=$ $x(t) / K, \tilde{y}(\tilde{t})=m y(t) / r K$, and letting $\delta=s / r, \beta=s h / m$, $a=A / K$, the system (2) is changed into a dimensionless form:

$$
\begin{aligned}
& \frac{d x}{d t}=x(1-x)-\frac{x}{a+x} y, \\
& \frac{d y}{d t}=y\left(\delta-\beta \frac{y}{x}\right) \\
& x(0)>0, y(0)>0 .
\end{aligned}
$$

In order to carry out integrated control of pests, we adopt strategies as follows.

(1) When the pest density $x(t)$ reached a lower level $x=$ $h_{1}$, we release natural enemies to control pests for low damage of insect pests on crops; that is,

$$
\begin{aligned}
& \Delta x(t)=0, \\
& \Delta y(t)=\lambda, x=h_{1}
\end{aligned}
$$

where $\lambda$ is amount of natural enemies $y(t)$ released one time.

(2) If the pest density $x(t)$ reached a higher level $x=h_{2}$, due to the fact that the damage of insect pests on crops is severe at this time, we effectively combine spraying pesticides with releasing natural enemies to control pests; that is,

$$
\begin{gathered}
\Delta x(t)=-p x(t), \\
\Delta y(t)=-q y(t)+\tau,
\end{gathered}
$$

Here, $\tau$ is the amount of natural enemies $y(t)$ released one time, $p, q$ are mortality rates of pests and natural enemies which die from spraying pesticides, and $p, q \in(0,1)$.
Synthesizing systems (3), (4), and (5), the following integrated pest management model is obtained:

$$
\begin{aligned}
& \frac{d x(t)}{d t}=x(t)(1-x(t))-\frac{x(t)}{a+x(t)} y(t), \\
& \frac{d y(t)}{d t}=y(t)\left(\delta-\beta \frac{y(t)}{x(t)}\right) \\
& x \neq h_{1}, h_{2}, \quad \text { or } \quad x=h_{1}, \quad y>\tilde{y} \\
& \Delta x(t)=0, \quad x=h_{1}, \quad y \leq \tilde{y} \\
& \Delta y(t)=\lambda, \quad x=h_{2} \\
& \Delta x(t)=-p x(t), \\
& \Delta y(t)=-q y(t)+\tau, \quad x(0)>0, \\
& x(0)>0, \quad y(t)
\end{aligned}
$$

where $x(t)$ denotes the population density of pests at time $t$ and $y(t)$ denotes the population density of natural enemies at time $t ; a, \delta, \beta$ are positive numbers; $p, q, \lambda, \tau$ are control parameters and positive numbers; the point $\left(h_{1}, \tilde{y}\right)$ is the intersection of the isoclinic line $y=(1-x)(x+a)$ and the straight line $x=h_{1}$.

\section{Preliminaries}

In order to analyze the dynamics of the system (6), we introduce the basic knowledge of the state impulsive differential equations.

Consider the state impulsive differential equation:

$$
\begin{aligned}
& \frac{d x(t)}{d t}=P(x, y), \\
& \frac{d y(t)}{d t}=Q(x, y), \\
& \Delta x(t)=\alpha(x, y), \quad(x, y) \notin M(x, y), \\
& \Delta y(t)=\beta(x, y), \quad
\end{aligned}
$$

where $P(x, y)$ and $Q(x, y)$ have order-one continuous partial derivatives.

Definition 1. The dynamic system which is formed by solution mapping of system (7) is called semicontinuous dynamic system, denoted by $(\Omega, f, \varphi, M)$, where $f$ is semicontinuous dynamical system mapping and $f: \Omega \rightarrow \Omega, \varphi(M)=N$, and in which $\varphi$ is pulse mapping. Here, $M(x, y)$ and $N(x, y)$ are straight or curved line in the plane. $M(x, y)$ is called impulsive set, and $N(x, y)$ is called corresponding image set.

In the system (6), let $M_{1}=\left\{(x, y) \mid x=h_{1}, 0<y \leq \tilde{y}\right\}$, and the image set corresponding to impulsive mapping (4) is $N_{1}=\varphi_{1}\left(M_{1}\right)=\left\{(x, y) \mid x=h_{1}, 0<y \leq \tilde{y}+\lambda\right\}$. Let $M_{2}=$ $\left\{(x, y) \mid x=h_{2}, y>0\right\}$, and the image set corresponding to impulsive mapping (5) is $N_{2}=\varphi_{2}\left(M_{2}\right)=\left\{(x, y) \mid x\left(t^{+}\right)=\right.$ $\left.(1-p) h_{2}, y\left(t^{+}\right)=(1-q) y(t)+\tau\right\}$.

Definition 2. Assume that impulsive set $M$ and image set $N$ are straight lines, the orbit $\Pi(A, t)$ of system (7) starting 
from point $A$ on $N$ hits $M$ at point $A_{1}$ and then jumps onto point $A_{1}^{+}$, then the function $f(A)=y_{A_{1}^{+}}-y_{A}$ is defined as a successor function about point $A$, and then point $A_{1}^{+}$is called successor point of $A$.

Lemma 3 (see $[10,11]$ ). Successor function is continuous.

Definition 4. If there exists a point $P_{0}$ on the image set $N$, and a constant $T>0$ such that $\Pi\left(P_{0}, T\right)=P \in M, \varphi(P)=P_{0} \in N$, then the orbit $\Pi\left(P_{0}, t\right)$ starting from $P_{0}$ is called an order-one periodic solution of the system (7).

Lemma 5 (Bendixson theorem of impulsive differential equations $[10,11])$. Assume that $G$ is a Bendixson region of system (7); if $G$ does not contain critical points of system (7), then system (7) contains a closed orbit in $G$.

For the system (6), from Lemma 5, the following conclusion is obtained.

Lemma 6. In system (6), if there exist points $A$ and $B$ on the image set $N$, such that the successor function satisfies $f(A) f(B)<0$, then there must exist an order-one periodic solution in system (6).

Lemma 7 (Analogue of Poincaré Criterion [3, 4]). Assume that $x=\xi(t), y=\eta(t)$ is the T-periodic solution of the following impulsive differential equations:

$$
\begin{aligned}
& \frac{d x(t)}{d t}=P(x, y), \\
& \frac{d y(t)}{d t}=Q(x, y), \\
& \Delta x(t)=\alpha(x, y), \Phi(x, y)=0, \\
& \Delta y(t)=\beta(x, y),
\end{aligned}
$$

where $P(x, y)$ and $Q(x, y)$ contain order-one continuous partial derivatives and $\Phi(x, y)$ is a sufficiently smooth function with $\operatorname{grad} \Phi(x, y) \neq 0$.

If the multiplier $\mu$ satisfies the condition $|\mu|<1$, then the periodic solution $(\xi(t), \eta(t))$ of the system (8) is orbitally asymptotically stable, where

$$
\begin{array}{r}
\mu=\prod_{j=1}^{n} \kappa_{j} \exp \left[\int _ { 0 } ^ { T } \left(\frac{\partial P(\xi(t), \eta(t))}{\partial x}\right.\right. \\
\left.\left.+\frac{\partial Q(\xi(t), \eta(t))}{\partial y}\right) d t\right],
\end{array}
$$

with

$$
\begin{aligned}
\kappa_{j}= & \left(\left(\frac{\partial \beta}{\partial y} \cdot \frac{\partial \Phi}{\partial x}-\frac{\partial \beta}{\partial x} \cdot \frac{\partial \Phi}{\partial y}+\frac{\partial \Phi}{\partial x}\right) P_{+}\right. \\
& \left.+\left(\frac{\partial \alpha}{\partial x} \cdot \frac{\partial \Phi}{\partial y}-\frac{\partial \alpha}{\partial y} \cdot \frac{\partial \Phi}{\partial x}+\frac{\partial \Phi}{\partial y}\right) Q_{+}\right) \\
& \times\left(\frac{\partial \Phi}{\partial x} P+\frac{\partial \Phi}{\partial y} Q\right)^{-1}
\end{aligned}
$$

and $P, Q, \partial \alpha / \partial x, \partial \alpha / \partial y, \partial \beta / \partial x, \partial \beta / \partial y, \partial \Phi / \partial x, \partial \Phi / \partial y$ are calculated at the point $\left(\xi\left(\tau_{j}\right), \eta\left(\tau_{j}\right)\right), P_{+}=P\left(\xi\left(\tau_{j}^{+}\right), \eta\left(\tau_{j}^{+}\right)\right)$, $Q_{+}=Q\left(\xi\left(\tau_{j}^{+}\right), \eta\left(\tau_{j}^{+}\right)\right)$, and $\tau_{j}(j \in N)$ is the time of the $j$ th jump.

\section{The Stability of System (6) without Impulsive Effect}

In the system (6), if $p=q=\lambda=\tau=0$, that is, the system without impulsive effect, the following system is obtained:

$$
\begin{aligned}
& \frac{d x}{d t}=x(1-x)-\frac{x}{a+x} y, \\
& \frac{d y}{d t}=y\left(\delta-\beta \frac{y}{x}\right), \\
& x(0)>0, y(0)>0 .
\end{aligned}
$$

If set $(x(t), y(t))$ is an arbitrary solution of the system (11) satisfying the initial conditions, then the following lemma is obtained.

Lemma 8. The solutions of the system (11) is bounded, which means $\exists T>0$ satisfies $0 \leq x(t) \leq 1$ and $0 \leq y(t) \leq \delta / \beta$ for $t \geq T$.

Obviously, the system (11) exhibits prey isocline $L_{1}: y=$ $(1-x)(x+a)$, predator isocline $L_{2}: y=(\delta / \beta) x$, nontrivial equilibrium points $E_{0}(1,0)$, and $E\left(x^{*}, y^{*}\right)$, and here;

$$
\begin{gathered}
x^{*}=\frac{(\beta-\delta-a \beta)+\sqrt{(\beta-\delta-a \beta)^{2}+4 a \beta^{2}}}{2 \beta}, \\
y^{*}=\frac{\delta}{\beta} x^{*} .
\end{gathered}
$$

Calculating the variational matrix of the equilibrium point in the system (11), we get

$$
J\left(E_{0}\right)=\left(\begin{array}{cc}
-1 & -\frac{1}{1+a} \\
0 & \delta
\end{array}\right) .
$$

Obviously, $E_{0}$ is saddle point. At $E$,

$$
J(E)=\left(\begin{array}{cc}
\frac{x^{*}}{x^{*}+a}\left(1-a-2 x^{*}\right) & -\frac{x^{*}}{x^{*}+a} \\
\frac{\delta^{2}}{\beta} & -\delta
\end{array}\right) .
$$

The characteristic equation of $J(E)$ is

$$
f(\lambda)=\lambda^{2}+p \lambda+q=0
$$

in which $p=\delta-\left(x^{*} /\left(x^{*}+a\right)\right)\left(1-a-2 x^{*}\right), q=\left(\delta x^{*} /\left(x^{*}+\right.\right.$ a) $)\left(\delta / \beta-\left(1-a-2 x^{*}\right)\right)$.

Thus,

$$
\begin{aligned}
\Delta=p^{2}-4 q= & \left(\delta-\frac{x^{*}}{x^{*}+a}\left(1-a-2 x^{*}\right)\right)^{2} \\
& -\frac{4 \delta x^{*}}{x^{*}+a}\left(\frac{\delta}{\beta}-\left(1-a-2 x^{*}\right)\right),
\end{aligned}
$$




$$
\begin{aligned}
\lambda_{1} \lambda_{2} & =\frac{\delta x^{*}}{x^{*}+a}\left(\frac{\delta}{\beta}-\left(1-a-2 x^{*}\right)\right) \\
& =\frac{\delta}{x^{*}+a}\left(x^{* 2}+a\right)>0, \\
\lambda_{1}+\lambda_{2} & =-\left(\delta-\frac{x^{*}}{x^{*}+a}\left(1-a-2 x^{*}\right)\right) \\
& =-\frac{2 x^{* 2}+(a-1+\delta) x^{*}+a \delta}{x^{*}+a} .
\end{aligned}
$$

Let $P(x)=2 x^{2}+(a-1+\delta) x+a \delta$, and then

$$
\begin{aligned}
\Delta & =\frac{P^{2}\left(x^{*}\right)}{\left(x^{*}+a\right)^{2}}-\frac{4 \delta\left(x^{* 2}+a\right)}{x^{*}+a} \\
& =\frac{1}{\left(x^{*}+a\right)^{2}}\left[P^{2}\left(x^{*}\right)-4 \delta\left(x^{* 2}+a\right)\left(x^{*}+a\right)\right] .
\end{aligned}
$$

Based on the above analysis, we can get the following conclusion.

Theorem 9. If $P\left(x^{*}\right)>0$, the positive equilibrium point $E\left(x^{*}\right.$, $\left.y^{*}\right)$ of the system (11) is locally asymptotically stable. Specially,

(1) if $\left(H_{1}\right): 0<P\left(x^{*}\right)<\left(4 \delta\left(x^{* 2}+a\right)\left(x^{*}+a\right)\right)^{1 / 2}, E\left(x^{*}\right.$, $\left.y^{*}\right)$ is a locally asymptotically stable focus,

(2) if $\left(H_{2}\right): P\left(x^{*}\right) \geqslant\left(4 \delta\left(x^{* 2}+a\right)\left(x^{*}+a\right)\right)^{1 / 2}, E\left(x^{*}, y^{*}\right)$ is a locally asymptotically stable node.

Next, we discuss the global stability of $E\left(x^{*}, y^{*}\right)$ about the system (11).

Theorem 10. If $\left(H_{3}\right): a+\delta \geq 1$ or $\left(H_{4}\right): 1-\sqrt{8 a \delta}<a+\delta<1$ is true, then the positive equilibrium $E\left(x^{*}, y^{*}\right)$ of the system (11) is globally asymptotically stable.

Proof. From $\left(H_{3}\right)$ or $\left(H_{4}\right)$, we have $P(x)=2 x^{2}+(a-1+$ $\delta) x+a \delta>0$ for $x>0$, and thus $P\left(x^{*}\right)>0$. Structure a Dulac function as follows:

$$
B(x, y)=\frac{x+a}{x y^{2}}, \quad x>0, y>0 .
$$

Let $f(x, y)=x(1-x)-(x /(a+x)) y, g(x, y)=y(\delta-\beta(y / x))$, and thus

$$
\begin{aligned}
\frac{\partial(f B)}{\partial x}+\frac{\partial(g B)}{\partial y} & =-\frac{1}{x y^{2}}\left[2 x^{2}+(a-1+\delta) x+a \delta\right] \\
& =-\frac{1}{x y^{2}} P(x) \leqslant 0
\end{aligned}
$$

By the Bendixson-Dulac theorem, there does not exist closed orbit of the system (11) around $E$. Based on Lemma 8 and Theorem 9, the positive equilibrium $E\left(x^{*}, y^{*}\right)$ is globally asymptotically stable.

Remark 11. If $\left(H_{1}\right),\left(H_{3}\right)$ or $\left(H_{1}\right),\left(H_{4}\right)$ are true, then $E\left(x^{*}, y^{*}\right)$ is a globally asymptotically stable focus.

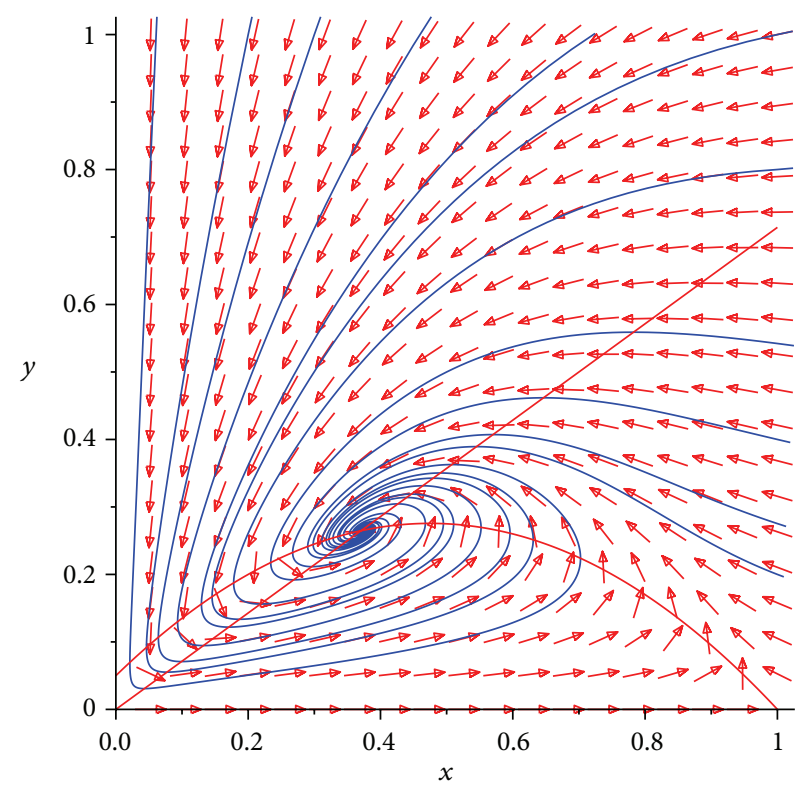

FIGURE 1: Illustration of vector graph of system (11), where $a=0.05$, $\delta=0.5, \beta=0.7$.

Remark 12. If $\left(H_{2}\right),\left(H_{3}\right)$ or $\left(H_{2}\right),\left(H_{4}\right)$ are true, then $E\left(x^{*}\right.$, $\left.y^{*}\right)$ is a globally asymptotically stable node.

Assume that $E\left(x^{*}, y^{*}\right)$ is globally asymptotically stable focal point of the system (11), and then the illustration of vector graph of the system is as follows (see Figure 1).

\section{The Geometric Analysis of System (6) with Two State Impulses}

In this section, we will discuss the existence and stability of periodic solution of system (6) only at focal point situation. So, we assume that the conditions $\left(H_{1}\right),\left(H_{3}\right)$ or $\left(H_{1}\right),\left(H_{4}\right)$ are true. According to the practical significance of the integrated pest management model, the condition $\left(H_{5}\right): h_{1}<(1-$ p) $h_{2}<h_{2}<x^{*}$ is always given as such. By the analysis of system (6), the curve $L_{1}: y=(1-x)(x+a)$ is $X$-isocline, and the line $L_{2}: y=(\delta / \beta) x$ is $Y$-isocline. Let points $P$, $Q, R$ be the intersection of the curve $L_{1}$ and lines $x=h_{1}$, $x=(1-p) h_{2}, x=h_{2}$, respectively. Obviously $E\left(x^{*}, y^{*}\right)$ is the intersection point of $L_{1}$ and $L_{2}$. From the previous discussion, we know that the first impulsive set is $M_{1}=\{(x, y) \mid x=$ $\left.h_{1}, 0<y \leq \tilde{y}\right\}$, and the image set corresponding to $M_{1}$ is $N_{1}=\left\{(x, y) \mid x=h_{1}, 0<y \leq \tilde{y}+\lambda\right\}$; the second impulsive set is $M_{2}=\left\{(x, y) \mid x=h_{2}, y>0\right\}$, and the image set corresponding to $M_{2}$ is $N_{2}=\left\{(x, y) \mid x\left(t^{+}\right)=\right.$ $\left.(1-p) h_{2}, y\left(t^{+}\right)=(1-q) y(t)+\tau\right\}$. The structure of the system can be shown as in Figure 2 .

Using successor function and geometric theory of impulsive differential equations and according to different positions of orbit initial points, the existence and stability of periodic solution of system (6) are discussed as follows.

4.1. The Initial Point on $N_{1}$. Let $C_{0}\left(h_{1}, y_{C_{0}}\right)$ be an initial point of the orbit of the system (6); if $y_{C_{0}}<\tilde{y}$, point $C_{0}$ is below 


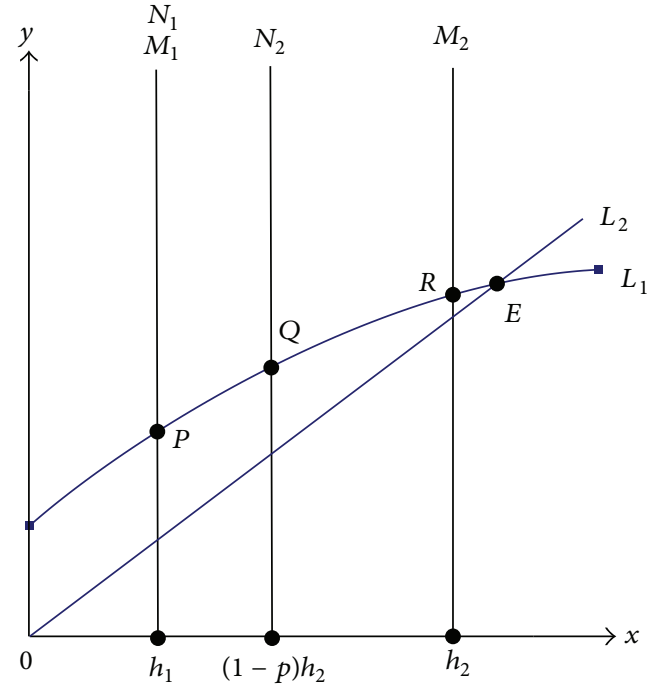

Figure 2: The structure graph of system (6).

point $P\left(h_{1}, \tilde{y}\right)$, then $C_{0} \in M_{1}\left(M_{1}\right.$ is impulsive set), and the image point $C_{0}^{+}$of $C_{0}$ must be above point $P$ with $n$ times impulses; therefore, we only need to discuss the cases of $y_{C_{0}}>$ $\tilde{y}$.

The orbit $\Pi\left(C_{0}, t\right)$ starting from $C_{0}\left(h_{1}, y_{C_{0}}\right)$ hits the impulsive set $M_{1}$ at point $C_{1}\left(h_{1}, y_{C_{1}}\right)$, and then $C_{1}$ jumps to point $C_{11}\left(h_{1}, y_{C_{11}}\right)$. If $y_{C_{11}}<\widetilde{y}, C_{11}$ continued to jump to point $C_{12}\left(h_{1}, y_{C_{12}}\right)$, and after $n$ times it reaches the point $C_{1 n}\left(h_{1}, y_{C_{1 n}}\right)$, where $y_{C_{11}}=y_{C_{1}}+\lambda, y_{C_{12}}=y_{C_{1}}+2 \lambda, \ldots$, $y_{C_{1 n}}=y_{C_{1}}+n \lambda$, and $\tilde{y}<y_{C_{1 n}}<\tilde{y}+\lambda$. The situation of point $C_{1 n}$ has three cases as follows.

(a) If $y_{C_{1 n}}=y_{C_{0}}$ (see Figure 3(a)), $C_{1 n}$ is coincident with $C_{0}$, then the curve $C_{0} C_{1} C_{11} \cdots C_{1 n}$ is closed orbit, and the system (6) exhibits a 1-periodic solution.

(b) If $y_{C_{1 n}}>y_{C_{0}}$ (see Figure $\left.3(\mathrm{~b})\right), C_{1 n}$ is above $C_{0}$; in this time, the successor function of $C_{0}$ satisfies $f\left(C_{0}\right)=y_{C_{1 n}}$ $y_{C_{0}}>0$. In the meantime, choose a point $D_{0}\left(h_{1}, y_{D_{0}}\right)$ on $N_{1}$ satisfying $y_{D_{0}}>\tilde{y}+\lambda$. The orbit $\Pi\left(D_{0}, t\right)$ starting from $D_{0}$ hits the impulsive set at point $D_{1}\left(h_{1}, y_{D_{1}}\right)$, and $D_{1}$ jumps sometimes to point $D_{1 m}\left(h_{1}, y_{D_{1 m}}\right)$, where $\tilde{y}<y_{D_{1 m}}<\tilde{y}+\lambda$. Thus, the successor function of $D_{0}$ satisfies $f\left(D_{0}\right)^{1 m}=y_{D_{1 m}}$ $y_{D_{0}}<0$.

According to Lemma 6, the system (6) exhibits a periodic solution, and the initial point of the periodic solution is between $C_{0}$ and $D_{0}$.

(c) If $y_{C_{1 n}}<y_{C_{0}}$ (see Figure $3(\mathrm{c})$ ), $C_{1 n}$ is below $C_{0}$; in this time, the successor function of $C_{0}$ is $f\left(C_{0}\right)=y_{C_{1 n}}-y_{C_{0}}<$ 0 . On the other hand, choose a point $D_{0}\left(h_{1}, y_{D_{0}}\right)$ on $N_{1}$ satisfying $\tilde{y}<y_{D_{0}}<\tilde{y}+\varepsilon$ ( $\varepsilon$ is a sufficiently small positive number). The orbit $\Pi\left(D_{0}, t\right)$ starting from $D_{0}$ hits the impulsive set $M_{1}$ at point $D_{1}\left(h_{1}, y_{D_{1}}\right)$, and $D_{1}$ jumps to point $D_{11}\left(h_{1}, y_{D_{11}}\right)$, where $y_{D_{11}}=y_{D_{1}}+\lambda$. As $D_{0}$ sufficiently closed to $P, D_{1}$ is sufficiently close to $P$, then $y_{D_{11}}=y_{D_{1}}+\lambda>y_{D_{0}}$. Thus, the successor function of $D_{0}$ satisfies $f\left(D_{0}\right)=y_{D_{1}}-$ $y_{D_{0}}>0$.

From Lemma 6, system (6) exhibits a 1-periodic solution. To sum up the above discussed, we get the following.
Theorem 13. If the initial point $C_{0}\left(h_{1}, y_{c_{0}}\right)$ of the orbit of the system (6) is on $N_{1}$ with $y_{C_{0}}>\tilde{y}$, then the system exhibits a 1-periodic solution.

Next, we will discuss the stability of the above periodic solutions.

Theorem 14. Let $(\xi(t), \eta(t))$ be the T-periodic solution of the system (6) with the initial point $C_{0}\left(h_{1}, \eta_{0}\right)$; the closed orbit corresponding to the periodic solution is the curve $C_{0} C_{1} C_{11} \cdots C_{1 n}$, if

$$
|\mu|=\left|\kappa \exp \left\{-\int_{0}^{T}\left(\xi(t)-\frac{\xi(t) \eta(t)}{(\xi(t)+a)^{2}}+\frac{\beta \eta(t)}{\xi(t)}\right) d t\right\}\right|<1,
$$

where

$$
\kappa=\frac{\eta_{0}-n \lambda}{\eta_{0}} \prod_{j=1}^{n} \frac{1-h_{1}-\left(1 /\left(h_{1}+a\right)\right)\left(\eta_{0}-(n-j) \lambda\right)}{1-h_{1}-\left(1 /\left(h_{1}+a\right)\right)\left(\eta_{0}-(n-j+1) \lambda\right)}
$$

then the periodic solution $(\xi(t), \eta(t))$ is orbitally asymptotically stable.

Proof. Let the orbit $\Pi\left(C_{0}, t\right)$ with the initial point $C_{0}\left(h_{1}, \eta_{0}\right)$ hit the impulsive set $M_{1}$ at $C_{1}(\xi(T), \eta(T))$, and then $C_{1}$ jumps to the point $C_{11}\left(\xi\left(\tau_{1}\right), \eta\left(\tau_{1}\right)\right)$. The $C_{11}$ continued to jump to point $C_{12}\left(\xi\left(\tau_{2}\right), \eta\left(\tau_{2}\right)\right)$, and at last, the image point $C_{12}$ reaches point $C_{1 n}\left(\xi\left(\tau_{n}\right), \eta\left(\tau_{n}\right)\right)$ with $n$ times pulses. Here, $\eta\left(\tau_{1}\right)=\eta(T)+\lambda, \eta\left(\tau_{2}\right)=\eta(T)+2 \lambda, \ldots, \eta\left(\tau_{n}\right)=\eta(T)+n \lambda$. For the $j$ th time impulse, obviously $\xi\left(\tau_{j}^{+}\right)=\xi\left(\tau_{j+1}\right), \eta\left(\tau_{j}^{+}\right)=$ $\eta\left(\tau_{j+1}\right)$. For the system (6), let $P(x, y)=x(1-x)-(x /(a+x)) y$, $Q(x, y)=y(\delta-\beta(y / x)), \alpha(x, y)=0, \beta(x, y)=\lambda, \Phi(x, y)=$ $x-h_{1}$, and $\xi(T)=h_{1}, \eta(T)=\eta_{0}-n \lambda$; therefore we have

$$
\begin{array}{cc}
\frac{\partial P}{\partial x}=1-2 x-\frac{a}{(a+x)^{2}} y, & \frac{\partial Q}{\partial y}=\delta-\frac{2 \beta y}{x} \\
\frac{\partial \alpha}{\partial x}=\frac{\partial \alpha}{\partial y}=0, & \frac{\partial \beta}{\partial x}=\frac{\partial \beta}{\partial y}=0, \\
\frac{\partial \Phi}{\partial x}=1, & \frac{\partial \Phi}{\partial y}=0 .
\end{array}
$$

According to Lemma 7, we get

$$
\begin{aligned}
\kappa_{j}= & \left(\left(\frac{\partial \beta}{\partial y} \cdot \frac{\partial \Phi}{\partial x}-\frac{\partial \beta}{\partial x} \cdot \frac{\partial \Phi}{\partial y}+\frac{\partial \Phi}{\partial x}\right) P_{+}\right. \\
& \left.+\left(\frac{\partial \alpha}{\partial x} \cdot \frac{\partial \Phi}{\partial y}-\frac{\partial \alpha}{\partial y} \cdot \frac{\partial \Phi}{\partial x}+\frac{\partial \Phi}{\partial y}\right) Q_{+}\right) \\
& \times\left(\frac{\partial \Phi}{\partial x} P+\frac{\partial \Phi}{\partial y} Q\right)^{-1}
\end{aligned}
$$


6

Abstract and Applied Analysis

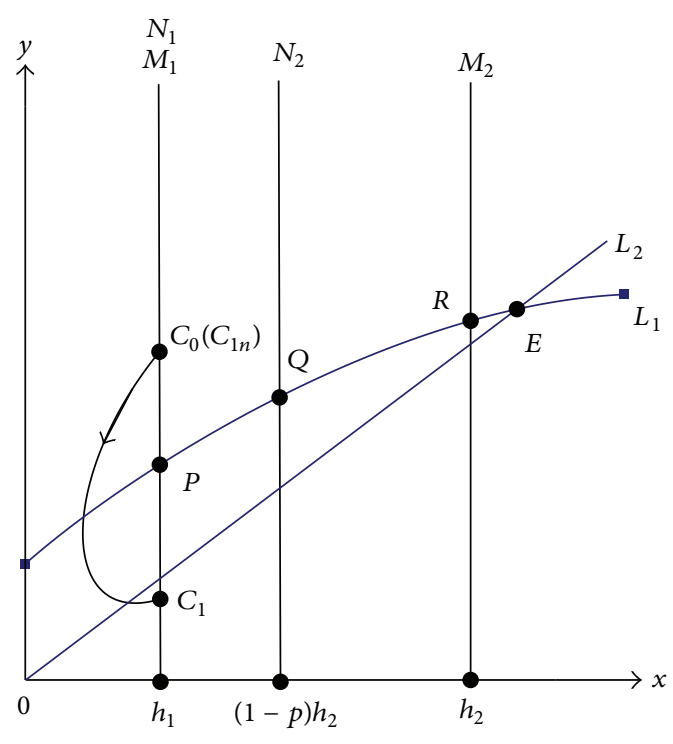

(a)

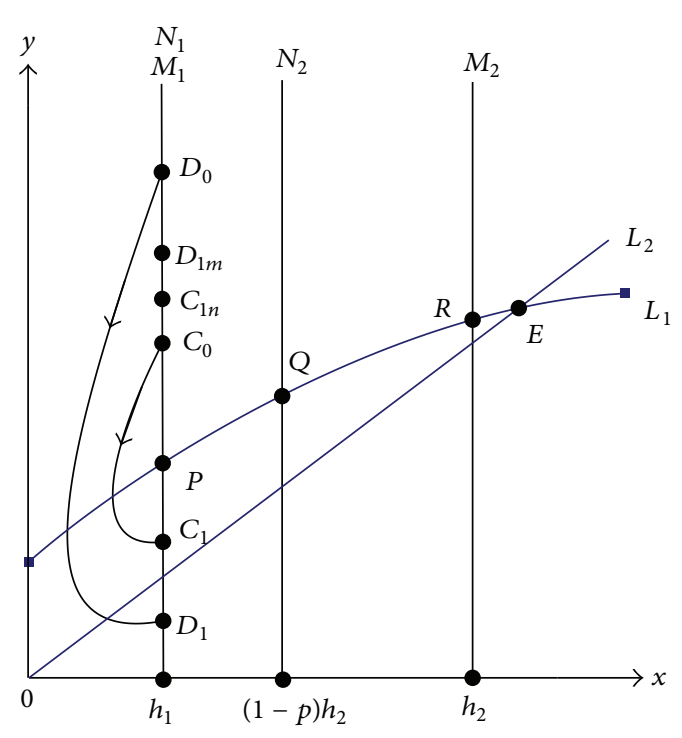

(b)

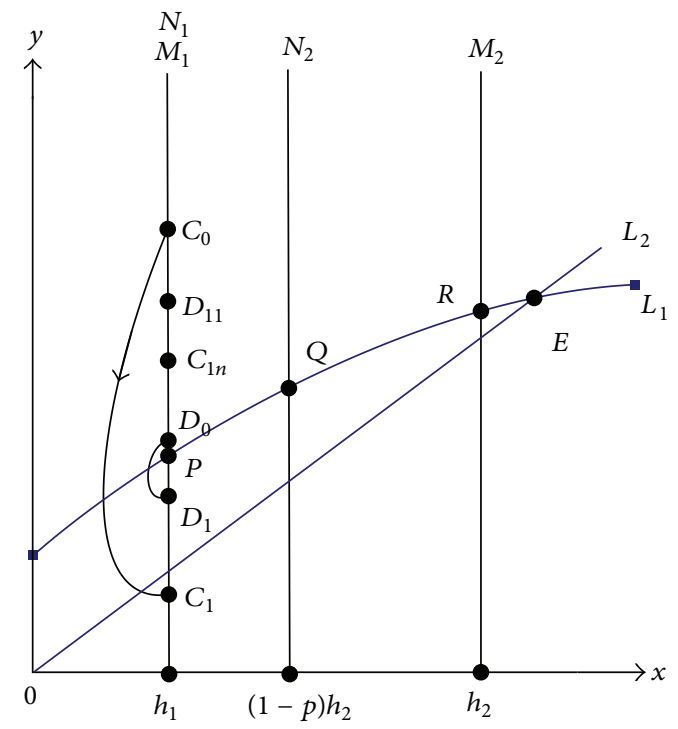

(c)

Figure 3: The orbit starting from the point $C_{0}$ on $N_{1}$.

$$
\begin{aligned}
& =\frac{P\left(\xi\left(\tau_{j}^{+}\right), \eta\left(\tau_{j}^{+}\right)\right)}{P\left(\xi\left(\tau_{j}\right), \eta\left(\tau_{j}\right)\right)} \\
= & \frac{P\left(h_{1}, \eta(T)+j \lambda\right)}{P\left(h_{1}, \eta(T)+(j-1) \lambda\right)} \\
= & \frac{1-h_{1}-\left(1 /\left(h_{1}+a\right)\right)\left(\eta_{0}-(n-j) \lambda\right)}{1-h_{1}-\left(1 /\left(h_{1}+a\right)\right)\left(\eta_{0}-(n-j+1) \lambda\right)} \\
= & 1-\frac{\lambda /\left(h_{1}+a\right)}{1-h_{1}-\left(1 /\left(h_{1}+a\right)\right)\left(\eta_{0}-(n-j+1) \lambda\right)}, \\
\mu & =\prod_{j=1}^{n} \kappa_{j} \exp \left[\int_{0}^{T}\left(\frac{\partial P}{\partial x}+\frac{\partial Q}{\partial y}\right) d t\right]
\end{aligned}
$$

$$
\begin{gathered}
=\prod_{j=1}^{n} \kappa_{j} \exp \left[\int_{0}^{T}\left(1-2 x-\frac{a}{(a+x)^{2}} y+\delta-\frac{2 \beta y}{x}\right) d t\right] \\
=\prod_{j=1}^{n} \kappa_{j} \exp \left\{\int _ { 0 } ^ { T } \left[\left(1-x-\frac{y}{x+a}\right)+\left(\delta-\frac{\beta y}{x}\right)\right.\right. \\
\left.\left.-x+\frac{x y}{(a+x)^{2}}-\frac{\beta y}{x}\right] d t\right\} \\
=\prod_{j=1}^{n} \kappa_{j} \exp \left\{\int_{0}^{T} \frac{d x}{x}+\int_{0}^{T} \frac{d y}{y}\right. \\
\left.-\int_{0}^{T}\left(x-\frac{x y}{(a+x)^{2}}+\frac{\beta y}{x}\right) d t\right\}
\end{gathered}
$$




$$
\begin{aligned}
= & \prod_{j=1}^{n} \kappa_{j} \frac{\eta_{0}-n \lambda}{\eta_{0}} \exp \left[-\int_{0}^{T}\left(x-\frac{x y}{(a+x)^{2}}+\frac{\beta y}{x}\right) d t\right] \\
= & \frac{\eta_{0}-n \lambda}{\eta_{0}} \\
& \times \prod_{j=1}^{n} \frac{1-h_{1}-\left(1 /\left(h_{1}+a\right)\right)\left(\eta_{0}-(n-j) \lambda\right)}{1-h_{1}-\left(1 /\left(h_{1}+a\right)\right)\left(\eta_{0}-(n-j+1) \lambda\right)} \\
& \times \exp \left[-\int_{0}^{T}\left(x-\frac{x y}{(a+x)^{2}}+\frac{\beta y}{x}\right) d t\right] \\
= & \kappa \exp \left\{-\int_{0}^{T}\left(\xi(t)-\frac{\xi(t) \eta(t)}{(\xi(t)+a)^{2}}+\frac{\beta \eta(t)}{\xi(t)}\right) d t\right\} .
\end{aligned}
$$

From Lemma 7, if $|\mu|=\mid \kappa \exp \left\{-\int_{0}^{T}(\xi(t)-(\xi(t) \eta(t) /(\xi(t)+\right.$ $\left.\left.\left.a)^{2}\right)+(\beta \eta(t) / \xi(t))\right) d t\right\} \mid<1$, then the periodic solution of the system (6) is orbitally asymptotically stable. This completes the proof.

Remark 15. If $1-h_{1}-\left(\eta_{0} /\left(h_{1}+a\right)\right)>0$ and $\beta \geq 1$, then the periodic solution with initial point $C_{0}\left(h_{1}, \eta_{0}\right)$ (where $\left.\eta_{0}>\tilde{y}\right)$ is orbitally asymptotically stable.

4.2. The Initial Point on $N_{2}$. Based on existence and uniqueness theorem of differential equations, there exists a unique point $Q_{0}\left((1-p) h_{2}, y_{Q_{0}}\right)$ on $N_{2}$ such that the orbit $\Pi\left(Q_{0}, t\right)$ starting from $Q_{0}$ is tangent to $N_{1}$ at point $P\left(h_{1}, \tilde{y}\right)$. Assume that $C_{0}\left((1-p) h_{2}, y_{C_{0}}\right)$ is the initial point of the orbit $\Pi\left(C_{0}, t\right)$ of system (6). Next, we will investigate the existence of periodic solution of the system with different positions of $C_{0}$ and $Q_{0}$. Three cases should be discussed.

Case I ( $y_{C_{0}}=y_{Q_{0}}$; see Figure 4$)$. The initial point $C_{0}$ is exactly $Q_{0}$.

The orbit $\Pi\left(Q_{0}, t\right)$ starting from $Q_{0}$ is tangent to $N_{1}$ at the point $P$, and through $N_{2}$ hit $M_{2}$ at the point $Q_{1}\left(h_{2}, y_{Q_{1}}\right)$, and then $Q_{1}$ jumps to $Q_{1}^{+}\left(x_{Q_{1}^{+}}, y_{Q_{1}^{+}}\right)$on $N_{2}$. According to (6), the following is obtained:

$$
x_{\mathrm{Q}_{1}^{+}}=(1-p) h_{2}, \quad y_{\mathrm{Q}_{1}^{+}}=(1-q) y_{\mathrm{Q}_{1}}+\tau \text {. }
$$

About the points $Q_{1}^{+}$and $Q_{0}$, there are the following three positional relations.

(a) If $Q_{1}^{+}$coincides with $Q_{0}: y_{Q_{1}^{+}}=y_{Q_{0}}$ (see Figure $4(\mathrm{a})$ ), then the curve $Q_{0} P Q_{1} Q_{1}^{+}$is closed orbit.

(b) If $Q_{1}^{+}$is below $Q_{0}: y_{Q_{1}^{+}}<y_{Q_{0}}$ (see Figure $4(\mathrm{~b})$ ), then the successor function of $Q_{0}$ satisfies $f\left(Q_{0}\right)=$ $y_{\mathrm{Q}_{1}^{+}}-y_{\mathrm{Q}_{0}}<0$. In the meantime, take a point $S_{0}((1-$ p) $\left.h_{2}, y_{S_{0}}\right)$ on $N_{2}$ satisfying $0<y_{S_{0}}<\varepsilon(\varepsilon>0$ small enough). The orbit $\Pi\left(S_{0}, t\right)$ starting from $S_{0}$ hits the impulsive $M_{2}$ at the point $S_{1}\left(h_{2}, y_{S_{1}}\right)$, and then $S_{1}$ jumps to the point $S_{1}^{+}\left(x_{S_{1}^{+}}, y_{S_{1}^{+}}\right)$, where $x_{S_{1}^{+}}=(1-p) h_{2}$,
$y_{S_{1}^{+}}=(1-q) y_{S_{1}}+\tau$. Obviously, the successor function of $S_{0}$ is $f\left(S_{0}\right)=y_{S_{1}^{+}}-y_{S_{0}}>0$. From Lemma 6 , the system (6) has an order one periodic solution, where the initial point of the periodic solution is between $Q_{0}$ and $S_{0}$.

(c) If $Q_{1}^{+}$is above $Q_{0}: y_{Q_{1}^{+}}>y_{Q_{0}}$ (see Figure $4(\mathrm{c})$ ), the system (6) does not have closed orbit in the area $\Omega_{1}=$ $\left\{(x, y) \mid h_{1}<x<h_{2}\right\}$ in this time.

Based on the discussion above, we get the following.

Theorem 16. Assume that the orbit $\Pi\left(Q_{0}, t\right)$ starting from $Q_{0}\left((1-p) h_{2}, y_{Q_{0}}\right)$ is tangent to $N_{1}$ at the point $P\left(h_{1}, \widetilde{y}\right)$ and hits the impulsive set $M_{2}$ at the point $Q_{1}\left(h_{2}, y_{Q_{1}}\right)$, the image point of $Q_{1}$ is $Q_{1}^{+}\left(x_{\mathrm{Q}_{1}^{+}}, y_{\mathrm{Q}_{1}^{+}}\right)$on $N_{2}$. If $y_{\mathrm{Q}_{1}^{+}} \leq y_{\mathrm{Q}_{0}}$, the system (6) has 1-periodic solution in the area $\Omega_{1}=\left\{(x, y) \mid h_{1}<x<h_{2}\right\}$.

Case II $\left(y_{\mathrm{C}_{0}}<y_{\mathrm{Q}_{0}}\right)$. The initial point $C_{0}$ is below $Q_{0}$.

The isocline $L_{1}: y=(1-x)(x+a)$ intersects with the phase set $N_{2}$ at the point $Q\left((1-p) h_{2}, y_{Q}\right)$, and $Q$ is below $Q_{0}$. In this case, we discuss the existence of periodic solution of the system (6) with the example $y_{C_{0}}=y_{Q}$ (see Figure 5).

The orbit $\Pi(Q, t)$ starting from $Q$ moves to the point $C_{1}\left(h_{2}, y_{C_{1}}\right)$ on the impulsive set $M_{2}$, and $C_{1}$ jumps onto $C_{1}^{+}\left(x_{C_{1}^{+}}, y_{C_{1}^{+}}\right)$on the image set $N_{2}$, and then

$$
\begin{aligned}
& y_{C_{0}}=y_{Q}=\left(1-(1-p) h_{2}\right)\left(a+(1-p) h_{2}\right), \\
& x_{C_{1}^{+}}=(1-p) h_{2}, \quad y_{C_{1}^{+}}=(1-q) y_{C_{1}}+\tau .
\end{aligned}
$$

(a) If $y_{C_{1}^{+}}=y_{Q}: C_{1}^{+}$coincide with $Q$ (see Figure 5(a)), the curve $Q C_{1} C_{1}^{+}$is the closed orbit of the system (6). (b) If $y_{Q}<y_{C_{1}^{+}} \leq y_{Q_{0}}: C_{1}^{+}$is between $Q$ and $Q_{0}$ (see Figure 5(b)), in this case the successor function of $Q$ is $f(Q)=y_{C_{1}^{+}}-y_{Q}>0$. On the other hand, consider the orbit $\Pi\left(Q_{0}, t\right)$ starting from $Q_{0}, \Pi\left(Q_{0}, t\right)$ hits the impulsive set $M_{2}$ at $Q_{1}\left(h_{2}, y_{Q_{1}}\right)$, and $Q_{1}$ jumps onto $\mathrm{Q}_{1}^{+}\left(x_{\mathrm{Q}_{1}^{+}}, y_{\mathrm{Q}_{1}^{+}}\right)$on $N_{2}$. Based on the existence and uniqueness theorem of differential equations, $Q_{1}$ must be below $C_{1}$, and $Q_{1}^{+}$must be below $C_{1}^{+}$. Thus $y_{Q_{1}^{+}}<y_{C_{1}^{+}} \leq y_{Q_{0}}$, and the successor function of $Q_{0}$ is $f\left(Q_{0}\right)=y_{Q_{1}^{+}}-y_{Q_{0}}<0$. From Lemma 6, the system (6) has 1-periodic solution, and the initial point of the periodic solution is between $Q_{0}$ and $Q$.

If $y_{C_{1}^{+}}>y_{Q_{0}}: C_{1}^{+}$is above $Q_{0}$, in this case the successor function of $Q$ is $f(Q)=y_{C_{1}^{+}}-y_{Q}>0$. There are two different cases (case (c) and case (d)).

(c) If $y_{C_{1}^{+}}>y_{Q_{0}}$ and $y_{Q_{1}^{+}} \leq y_{Q_{0}}: Q_{1}^{+}$is below $Q_{0}$ (see Figure 5(c)), $f\left(Q_{0}\right)=y_{Q_{1}^{+}}-y_{Q_{0}} \leq 0$, the system (6) has closed orbit.

(d) If $y_{C_{1}^{+}}>y_{Q_{0}}$ and $y_{Q_{1}^{+}}>y_{Q_{0}}: Q_{1}^{+}$is above $Q_{0}$ (see Figure 5(d)), the system (6) does not have closed orbit in the area $\Omega_{1}=\left\{(x, y) \mid h_{1}<x<h_{2}\right\}$. 


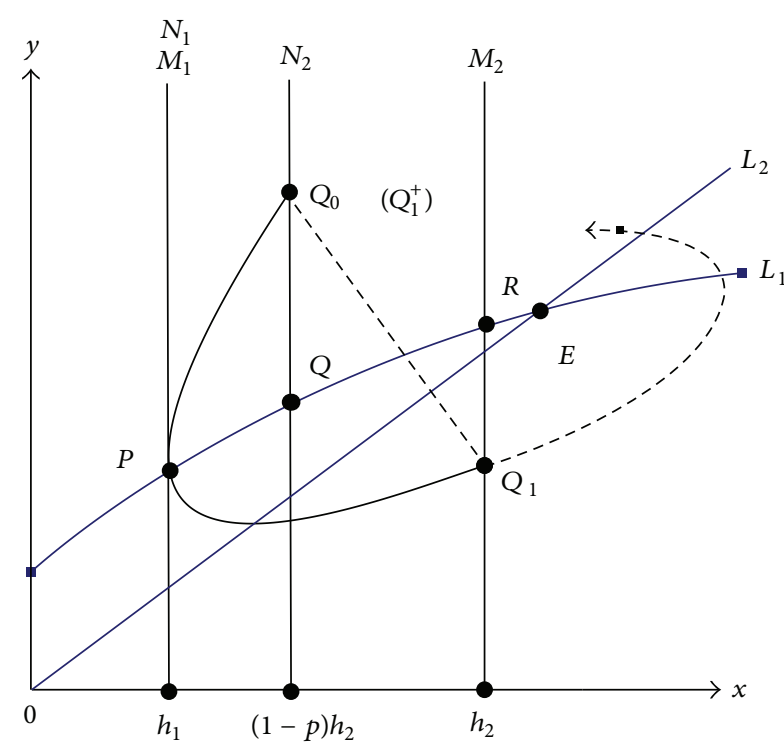

(a)

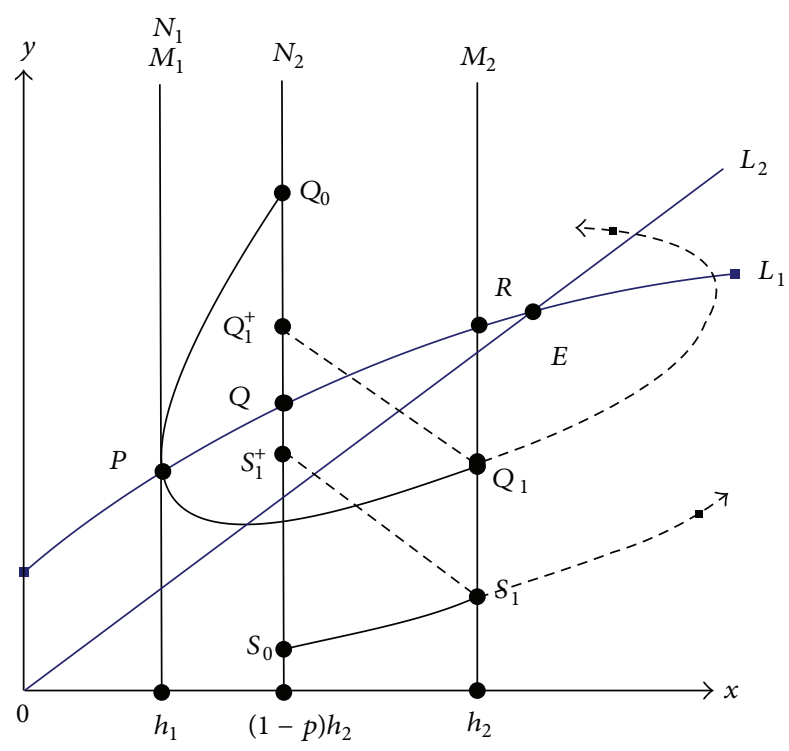

(b)

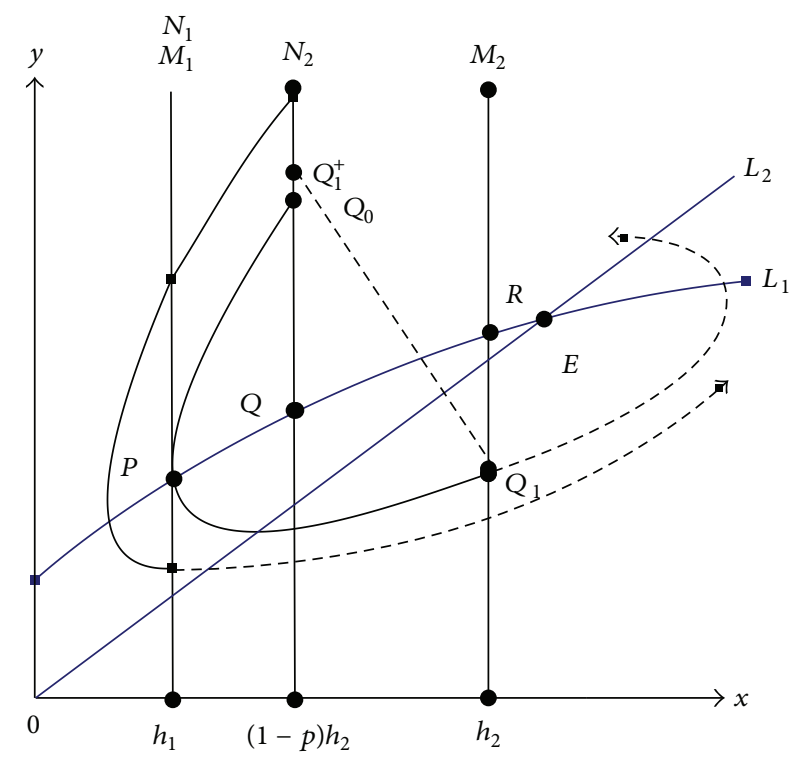

(c)

FIgure 4: The initial point $C_{0}$ on $N_{2}$ (Case I: $y_{C_{0}}=y_{\mathrm{Q}_{0}}$ ).

(e) If $y_{C_{1}^{+}}<y_{Q}: C_{1}^{+}$is below $Q$ (see Figure 5(e)), in this case the successor function of $Q$ is $f(Q)=$ $y_{C_{1}^{+}}-y_{\mathrm{Q}}<0$. On the other hand, take a point $D_{0}((1-$ p) $h_{2}, y_{D_{0}}$ ) from $N_{2}$ satisfying that $y_{D_{0}}$ is sufficiently small number, which is $0<y_{D_{0}}<\varepsilon(\varepsilon>0$ small enough). The orbit $\Pi\left(D_{0}, t\right)$ starting from $D_{0}$ moves to $D_{1}\left(h_{2}, y_{D_{1}}\right)$ on the impulsive set $M_{2}$, and $D_{1}$ jumps onto $D_{1}^{+}\left(x_{D_{1}^{+}}, y_{D_{1}^{+}}\right)$on $N_{2}$, where $x_{D_{1}^{+}}=(1-p) h_{2}$, $y_{D_{1}^{+}}=(1-q) y_{D_{1}}+\tau$. Obviously, the successor function of $D_{0}$ is $f\left(D_{0}\right)=y_{D_{1}^{+}}-y_{D_{0}}>0$. From Lemma 6 , the system (6) has closed orbit.

Based on the discussion above, we get the following.

Theorem 17. Assume that the orbit $\Pi(Q, t)$ starting from $Q\left((1-p) h_{2}, y_{Q}\right)$ hits the impulsive set $M_{2}$ at $C_{1}\left(h_{2}, y_{C_{1}}\right)$, and $C_{1}$ jumps onto $C_{1}^{+}\left(x_{C_{1}^{+}}, y_{C_{1}^{+}}\right)$on $N_{2}$. The orbit $\Pi\left(Q_{0}, t\right)$ starting from the point $Q_{0}\left((1-p) h_{2}, y_{Q_{0}}\right)$ is tangent to $N_{1}$ at $P\left(h_{1}, \tilde{y}\right)$, 


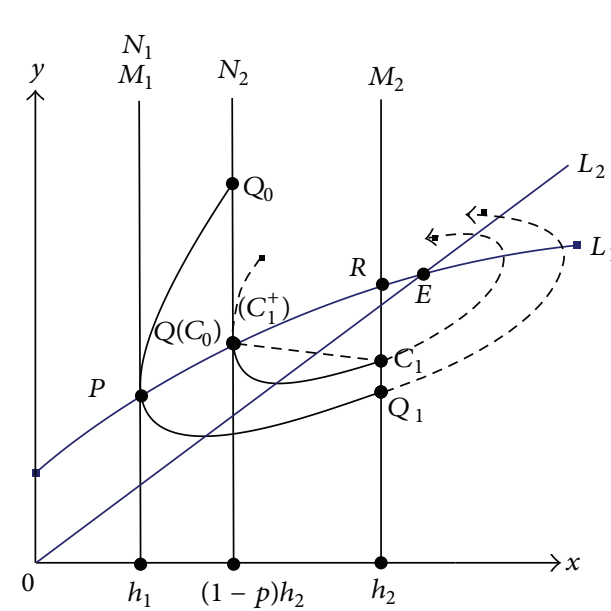

(a)

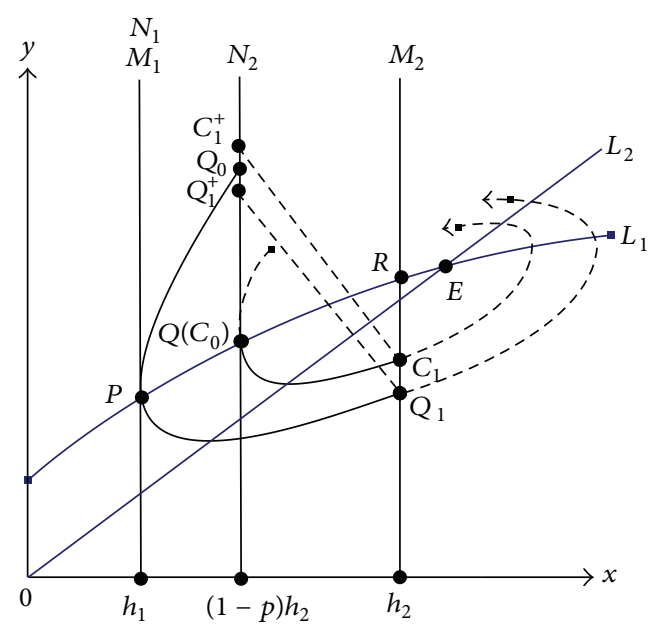

(c)

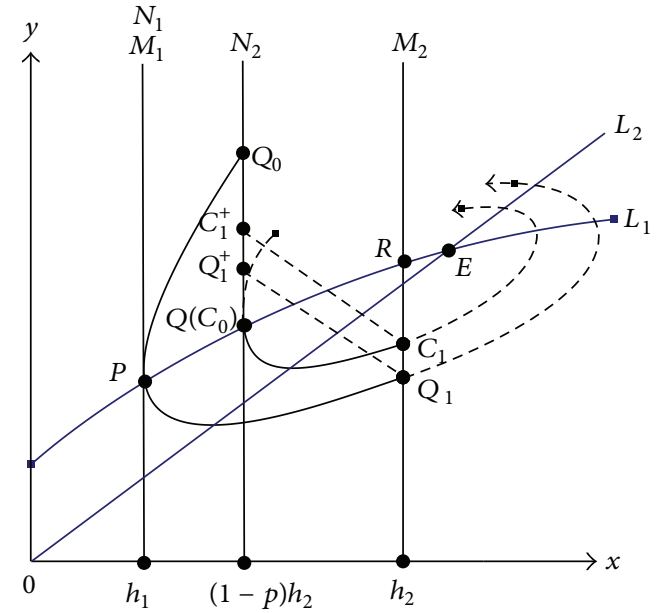

(b)

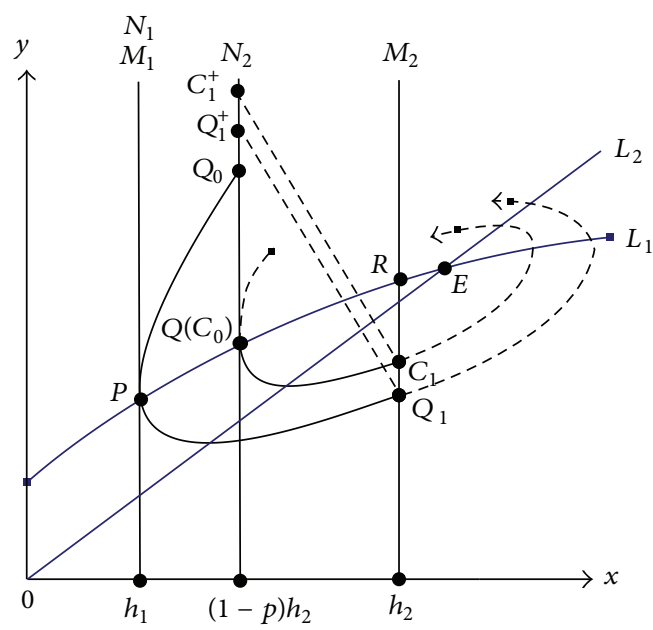

(d)

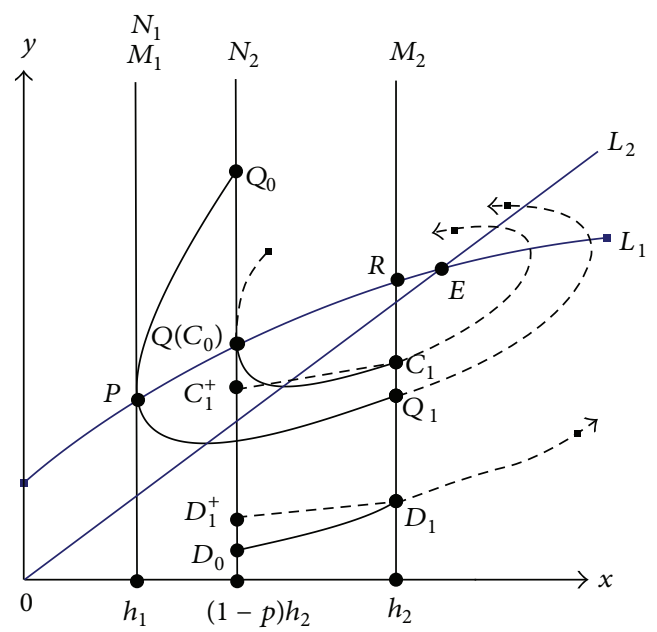

(e)

FIGURE 5: The initial point $C_{0}$ on $N_{2}$ (Case II: $y_{C_{0}}<y_{Q_{0}}$ ). 


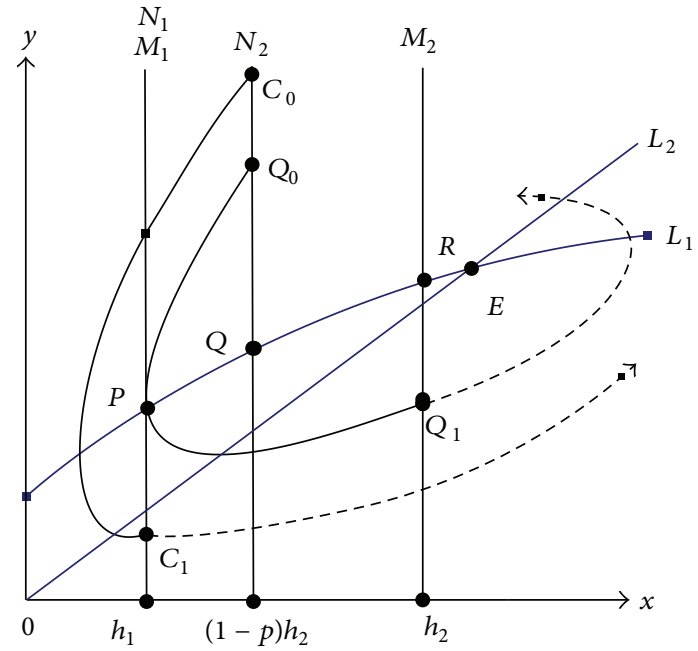

FIgure 6: The initial point $C_{0}$ on $N_{2}$ (Case III: $y_{C_{0}}>y_{\mathrm{Q}_{0}}$ ).

and hitting the impulsive set $M_{2}$ at $Q_{1}\left(h_{2}, y_{Q_{1}}\right)$, the image point of $Q_{1}$ is $Q_{1}^{+}\left(x_{Q_{1}^{+}}, y_{Q_{1}^{+}}\right)$. Then one has the following.

(1) If $y_{C_{1}^{+}} \leq y_{Q_{0}}$, the system (6) has 1-periodic solution.

(2) If $y_{C_{1}^{+}}>y_{Q_{0}}$ and $y_{Q_{1}^{+}} \leq y_{Q_{0}}$, the system (6) has 1periodic solution.

Case III ( $y_{C_{0}}>y_{\mathrm{Q}_{0}}$; see Figure 6). The initial point $C_{0}$ is above $Q_{0}$.

In this case, the orbit $\Pi\left(C_{0}, t\right)$ starting from $C_{0}$ goes through the isocline $L_{1}$ from the left of the line $x=h_{1}$, hitting the impulsive set $M_{1}$ at $C_{1}\left(h_{1}, y_{C_{1}}\right)$. The same conclusion can be made as in Section 4.1.

Next, we discuss the stability of the periodic solution with the initial point on $N_{2}$.

Theorem 18. Assume that $(\xi(t), \eta(t))$ is the T-periodic solution of the system (6) with initial point $C_{0}\left((1-p) h_{2}, \eta_{0}\right)$; and if

$$
|\mu|=\left|\kappa \exp \left\{-\int_{0}^{T}\left(\xi(t)-\frac{\xi(t) \eta(t)}{(\xi(t)+a)^{2}}+\frac{\beta \eta(t)}{\xi(t)}\right) d t\right\}\right|<1,
$$

where

$$
\kappa=\frac{\eta_{0}-\tau}{\eta_{0}} \cdot \frac{1-(1-p) h_{2}-\left(\eta_{0} /\left((1-p) h_{2}+a\right)\right)}{1-h_{2}-\left(\left(\eta_{0}-\tau\right) /\left((1-q)\left(h_{2}+a\right)\right)\right)},
$$

the periodic solution $(\xi(t), \eta(t))$ is orbitally asymptotically stable.

Proof. Assume that the periodic orbit $\Pi\left(C_{0}, t\right)$ starting from the point $C_{0}\left((1-p) h_{2}, \eta_{0}\right)$ moves to the point $C_{1}(\xi(T), \eta(T))$ on impulsive set $M_{2}$, and $C_{1}$ jumps onto the point $C_{1}^{+}\left(\xi\left(T^{+}\right), \eta\left(T^{+}\right)\right)$on $N_{2}$. Therefore, $\Pi\left(C_{0}, T\right)=C_{1}$, $C_{1}^{+}=\varphi_{2}\left(C_{1}\right)=C_{0}, \xi\left(T^{+}\right)=(1-p) \xi(T), \eta\left(T^{+}\right)=(1-q) \eta(T)+$ $\tau$.
Compared with the system (6), we get

$$
\begin{gathered}
P(x, y)=x(1-x)-\frac{x}{a+x} y, \\
Q(x, y)=y\left(\delta-\beta \frac{y}{x}\right), \\
\alpha(x, y)=-p x, \quad \beta(x, y)=-q y+\tau, \\
\Phi(x, y)=x-h_{2}, \quad \xi(T)=h_{2}, \quad \eta(T)=\frac{\eta_{0}-\tau}{1-q}, \\
\frac{\partial P}{\partial x}=1-2 x-\frac{a}{(a+x)^{2}} y, \quad \frac{\partial Q}{\partial y}=\delta-\frac{2 \beta y}{x}, \\
\frac{\partial \alpha}{\partial x}=-p, \quad \frac{\partial \alpha}{\partial y}=0, \quad \frac{\partial \beta}{\partial x}=0, \\
\frac{\partial \beta}{\partial y}=-q, \quad \frac{\partial \Phi}{\partial x}=1, \quad \frac{\partial \Phi}{\partial y}=0 .
\end{gathered}
$$

Thus,

$$
\begin{aligned}
& \kappa_{1}=\left(\left(\frac{\partial \beta}{\partial y} \cdot \frac{\partial \Phi}{\partial x}-\frac{\partial \beta}{\partial x} \cdot \frac{\partial \Phi}{\partial y}+\frac{\partial \Phi}{\partial x}\right) P_{+}\right. \\
& \left.+\left(\frac{\partial \alpha}{\partial x} \cdot \frac{\partial \Phi}{\partial y}-\frac{\partial \alpha}{\partial y} \cdot \frac{\partial \Phi}{\partial x}+\frac{\partial \Phi}{\partial y}\right) Q_{+}\right) \\
& \times\left(\frac{\partial \Phi}{\partial x} P+\frac{\partial \Phi}{\partial y} Q\right)^{-1} \\
& =\frac{(1-q) P\left(\xi\left(T^{+}\right), \eta\left(T^{+}\right)\right)}{P(\xi(T), \eta(T))} \\
& =((1-p)(1-q) \\
& \left.\times\left(1-(1-p) h_{2}-\frac{\eta_{0}}{(1-p) h_{2}+a}\right)\right) \\
& \times\left(1-h_{2}-\frac{\eta_{0}-\tau}{(1-q)\left(h_{2}+a\right)}\right)^{-1}, \\
& \mu=\kappa_{1} \exp \left[\int_{0}^{T}\left(\frac{\partial P}{\partial x}+\frac{\partial Q}{\partial y}\right) d t\right] \\
& =\kappa_{1} \exp \left[\int_{0}^{T}\left(1-2 x-\frac{a}{(a+x)^{2}} y+\delta-\frac{2 \beta y}{x}\right) d t\right] \\
& =\kappa_{1} \exp \left\{\int _ { 0 } ^ { T } \left[\left(1-x-\frac{y}{x+a}\right)+\left(\delta-\frac{\beta y}{x}\right)\right.\right. \\
& \left.\left.-x+\frac{x y}{(a+x)^{2}}-\frac{\beta y}{x}\right] d t\right\}
\end{aligned}
$$




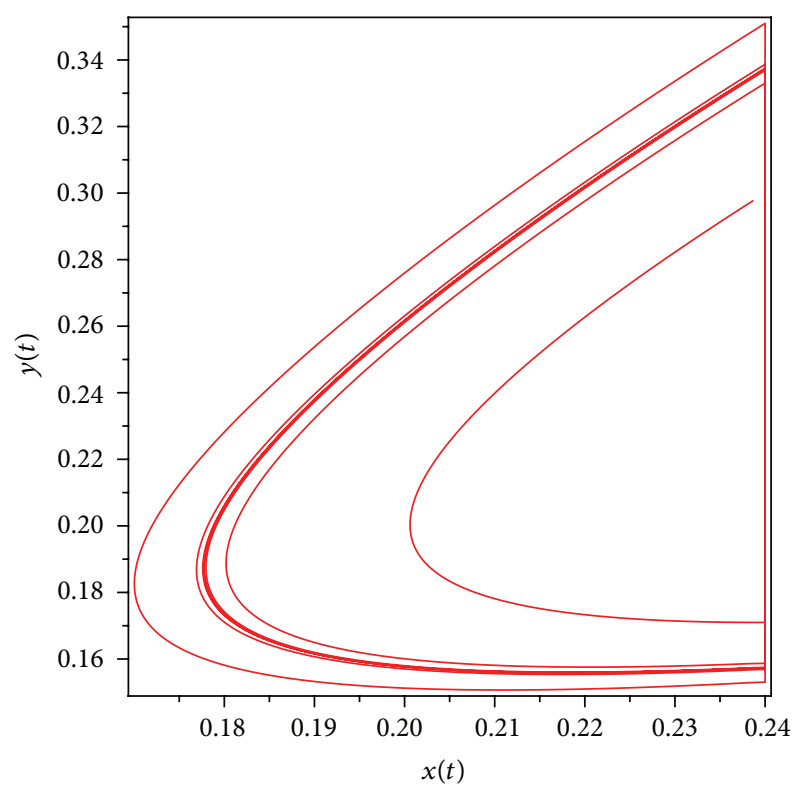

(a) Phase diagram of system (30)

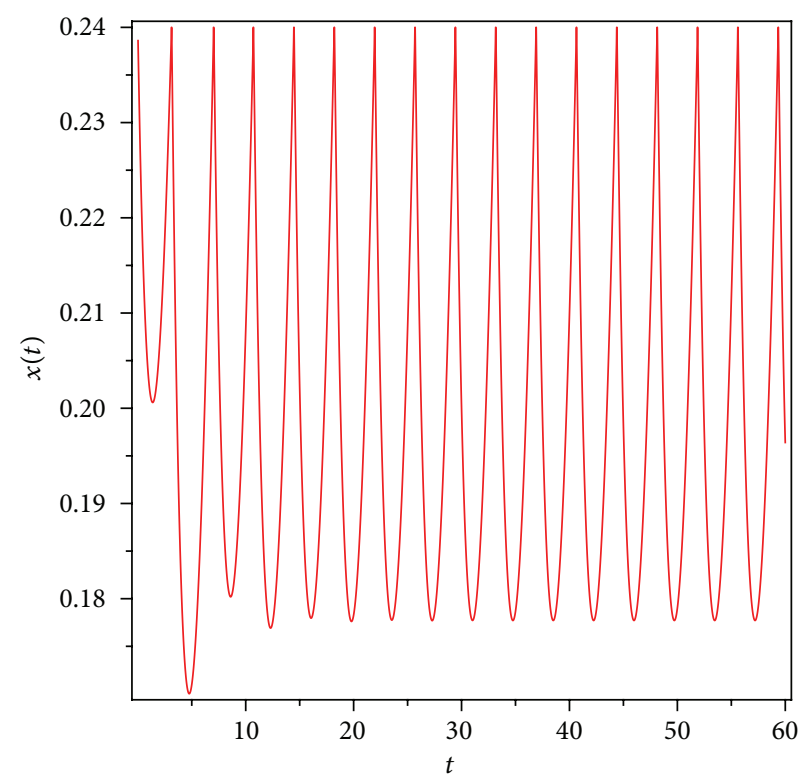

(b) Time series of system (30)

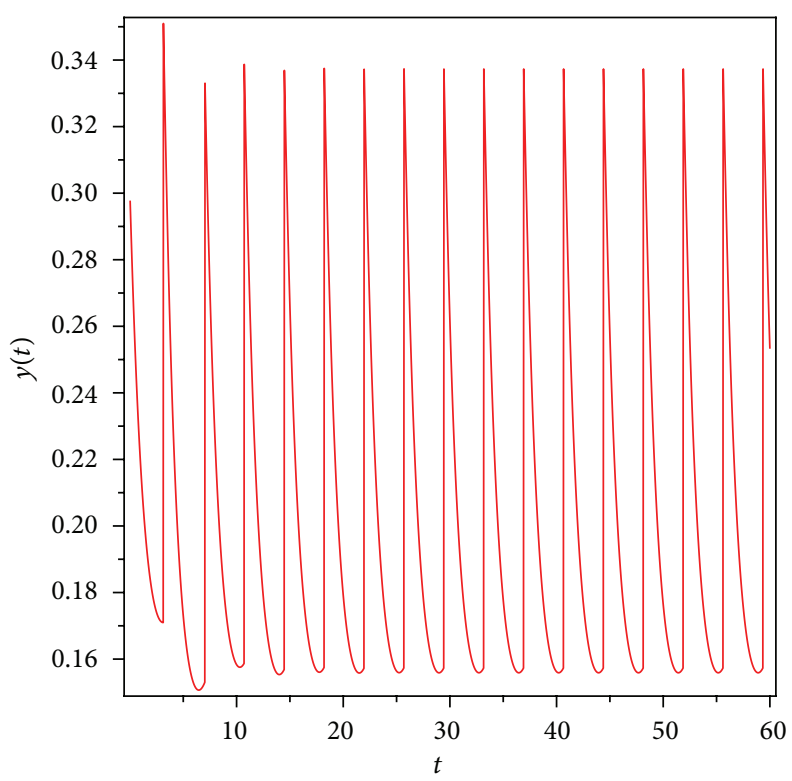

(c) Time series of system (30)

FIgURE 7: The periodic solution corresponding to Figure 3.

$$
\begin{aligned}
= & \kappa_{1} \exp \left\{\int_{0}^{T} \frac{d x}{x}+\int_{0}^{T} \frac{d y}{y}-\int_{0}^{T}\left(x-\frac{x y}{(a+x)^{2}}+\frac{\beta y}{x}\right) d t\right\} \\
= & \frac{(1-p)(1-q)\left(1-(1-p) h_{2}-\left(\eta_{0} /\left((1-p) h_{2}+a\right)\right)\right)}{1-h_{2}-\left(\left(\eta_{0}-\tau\right) /\left((1-q)\left(h_{2}+a\right)\right)\right)} \\
& \quad \exp \left\{\int_{0}^{T} \frac{d x}{x}+\int_{0}^{T} \frac{d y}{y}-\int_{0}^{T}\left(x-\frac{x y}{(a+x)^{2}}+\frac{\beta y}{x}\right) d t\right\} \\
= & \frac{\eta_{0}-\tau}{\eta_{0}} \cdot \frac{1-(1-p) h_{2}-\left(\eta_{0} /\left((1-p) h_{2}+a\right)\right)}{1-h_{2}-\left(\left(\eta_{0}-\tau\right) /\left((1-q)\left(h_{2}+a\right)\right)\right)}
\end{aligned}
$$$$
\times \exp \left[-\int_{0}^{T}\left(x-\frac{x y}{(a+x)^{2}}+\frac{\beta y}{x}\right) d t\right]
$$

From Lemma 7, if $|\mu|=\mid \kappa \exp \left\{-\int_{0}^{T}(\xi(t)-(\xi(t) \eta(t) \mid\right.$ $\left.\left.\left.(\xi(t)+a)^{2}\right)+(\beta \eta(t) / \xi(t))\right) d t\right\} \mid<1$, then the periodic solution 


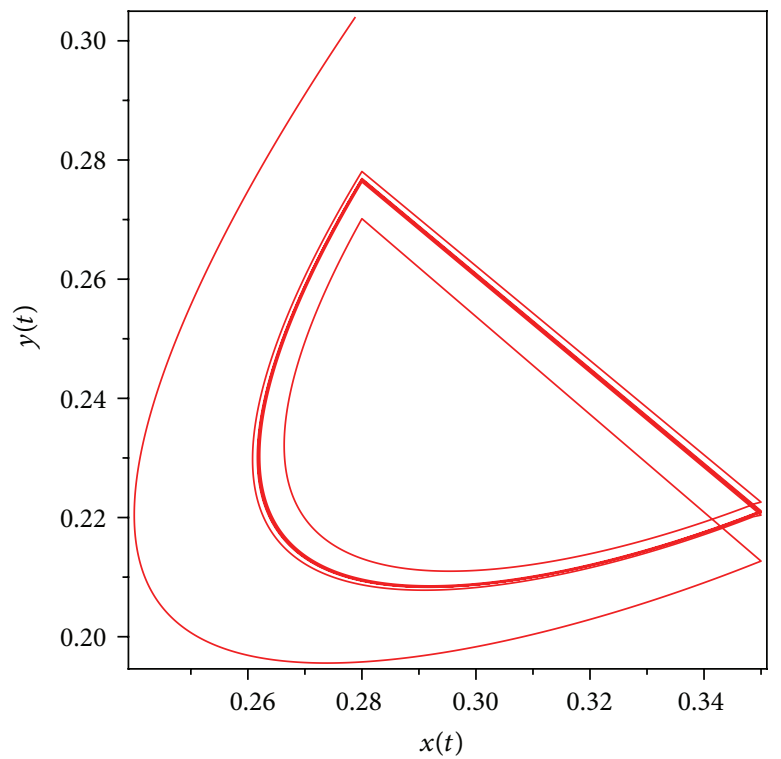

(a) Phase diagram of system (30)

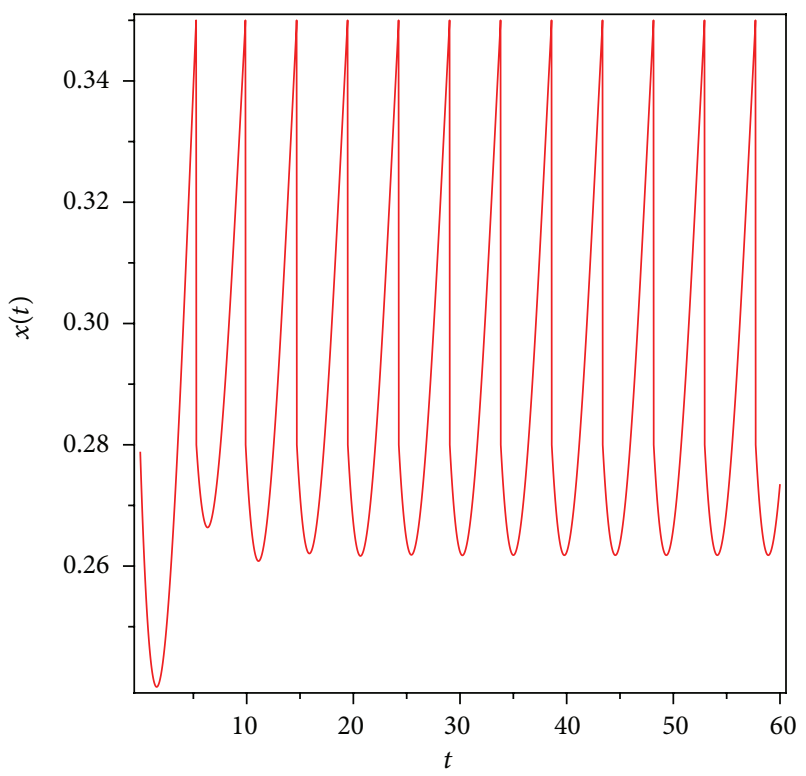

(b) Time series of system (30)

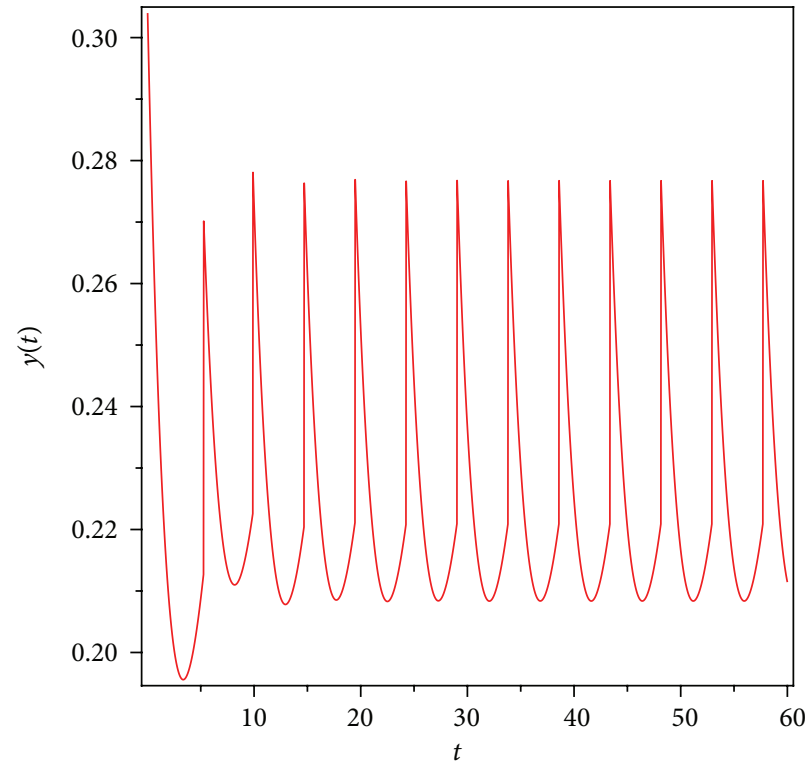

(c) Time series of system (30)

FiguRE 8: There exists a 1-periodic solution in the area $\Omega_{1}=\left\{(x, y) \mid h_{1}<x<h_{2}\right\}$ corresponding to Figure 4(b).

of system (6) is orbitally asymptotically stable. This completes the proof.

Remark 19. If $\mid\left(\left(1-(1-p) h_{2}\right)-\left(\eta_{0} /\left((1-p) h_{2}+a\right)\right)\right) /(1-$ $\left.h_{2}-\left(\left(\eta_{0}-\tau\right) /\left((1-q)\left(h_{2}+a\right)\right)\right)\right) \mid \leq 1$ and $\beta \geq 1$, the periodic solution of system (6) with initial point $C_{0}\left((1-p) h_{2}, \eta_{0}\right)$ is orbitally asymptotically stable.

\section{Example and Numerical Simulation}

In this part, we use numerical simulation to confirm the conclusion obtained above. Let $a=0.05, \delta=0.5, \beta=0.7$, $h_{1}=0.24, h_{2}=0.35, \lambda=0.18, p=0.2, q=0.2$. By calculation, we obtain $\tilde{y}=0.22, P(0.24,0.22), Q(0.28,0.2376)$ and $R(0.35,0.26)$. Then, we have an example as follows:

$$
\begin{aligned}
& \frac{d x(t)}{d t}=x(t)(1-x(t))-\frac{x(t)}{0.05+x(t)} y(t), \\
& \frac{d y(t)}{d t}=y(t)\left(0.5-0.7 \frac{y(t)}{x(t)}\right), \\
& x \neq 0.24,0.35 \quad \text { or } \quad x=0.24, \quad y>0.22, \\
& \Delta x(t)=0, \quad x=0.24, \quad y \leq 0.22, \\
& \Delta y(t)=0.18, \quad x \quad
\end{aligned}
$$




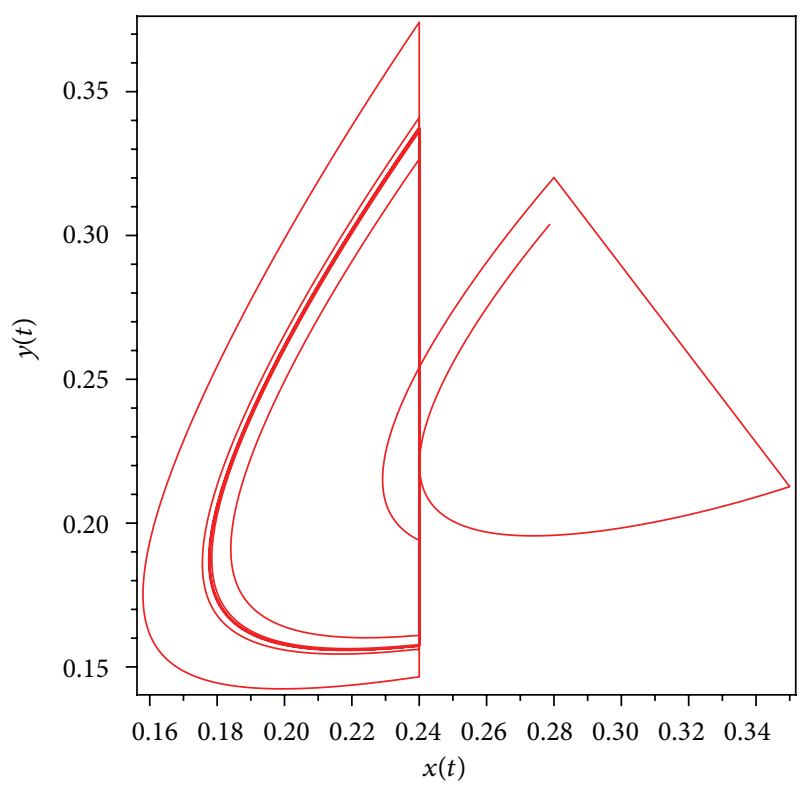

(a) Phase diagram of system (30)

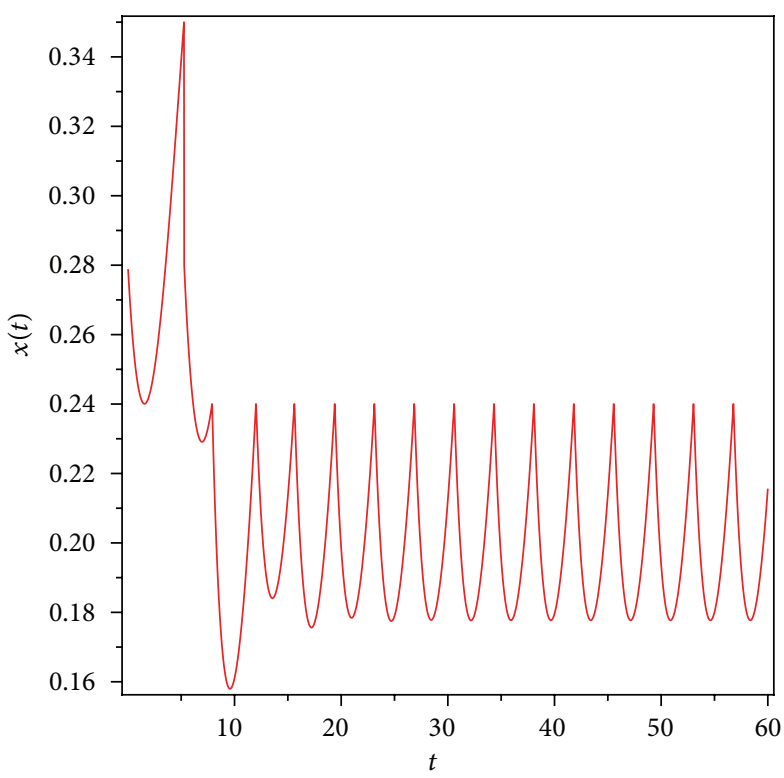

(b) Time series of system (30)

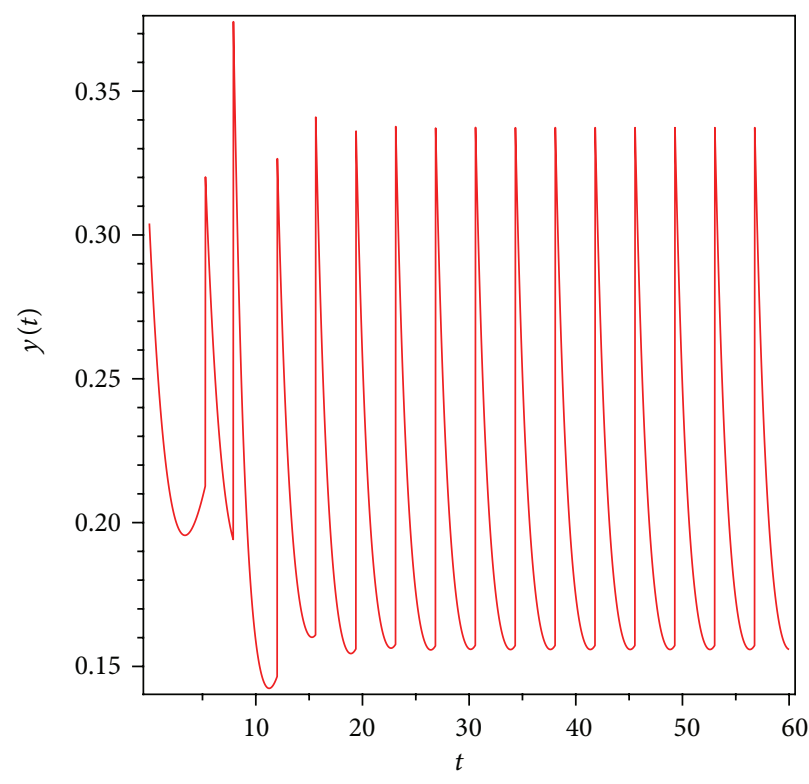

(c) Time series of system (30)

FIGURE 9: 1-periodic solution in the area $\Omega_{1}=\left\{(x, y) \mid h_{1}<x<h_{2}\right\}$ corresponding to Figure 4(c) does not exist.

$$
\begin{gathered}
\Delta x(t)=-0.2 x(t), \\
\Delta y(t)=-0.2 y(t)+\tau, \quad x=0.35, \\
x(0)>0, \quad y(0)>0 .
\end{gathered}
$$

Case 1. Let $\tau=0.15$, and the initial point is $(0.24,0.3)$. From Figure 7 corresponding to Figure 3, the system exhibits a 1periodic solution.

Case 2. Let $\tau=0.1$, then $(0.28,0.3056)$ is the initial point. From Figure 8, which corresponds to Figure 4(b), the system exhibits a 1-periodic solution in the area $\Omega_{1}=\left\{(x, y) \mid h_{1}<\right.$ $\left.x<h_{2}\right\}$.

Case 3. Let $\tau=0.15$, we get the initial point $(0.28,0.3056)$. It is easy to find that the system has no 1-periodic solution in the area $\Omega_{1}=\left\{(x, y) \mid h_{1}<x<h_{2}\right\}$ from Figure 9 which corresponds to Figure 4(c).

Case 4. Let $\tau=0.1$, the initial point $(0.28,0.2376)$ is obtained. From Figure 10 corresponding to Figure 5(b), in the area $\Omega_{1}=$ $\left\{(x, y) \mid h_{1}<x<h_{2}\right\}$, the system exhibits a 1-periodic solution. 


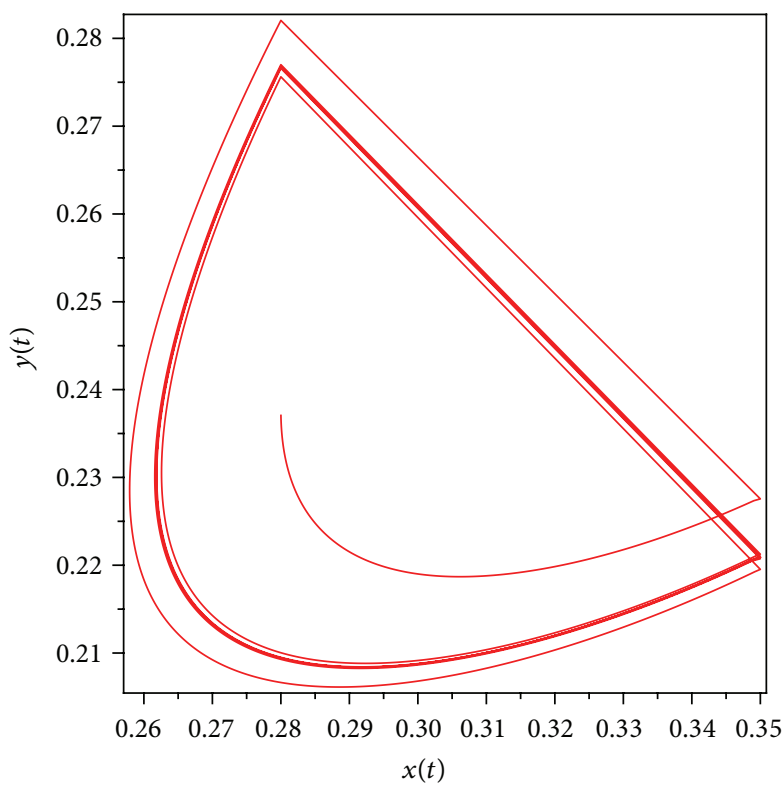

(a) Phase diagram of system (30)

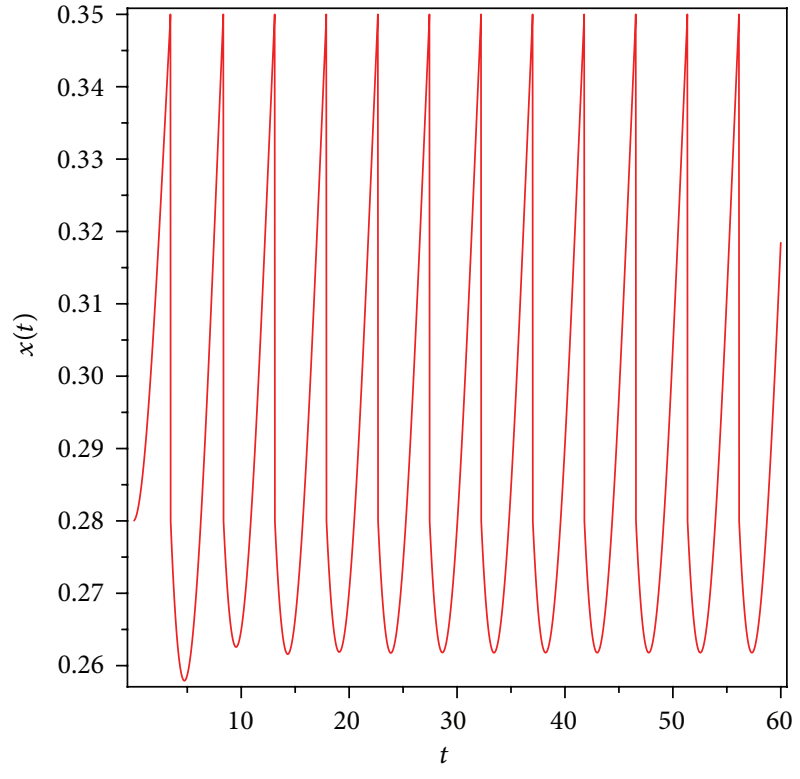

(b) Time series of system (30)

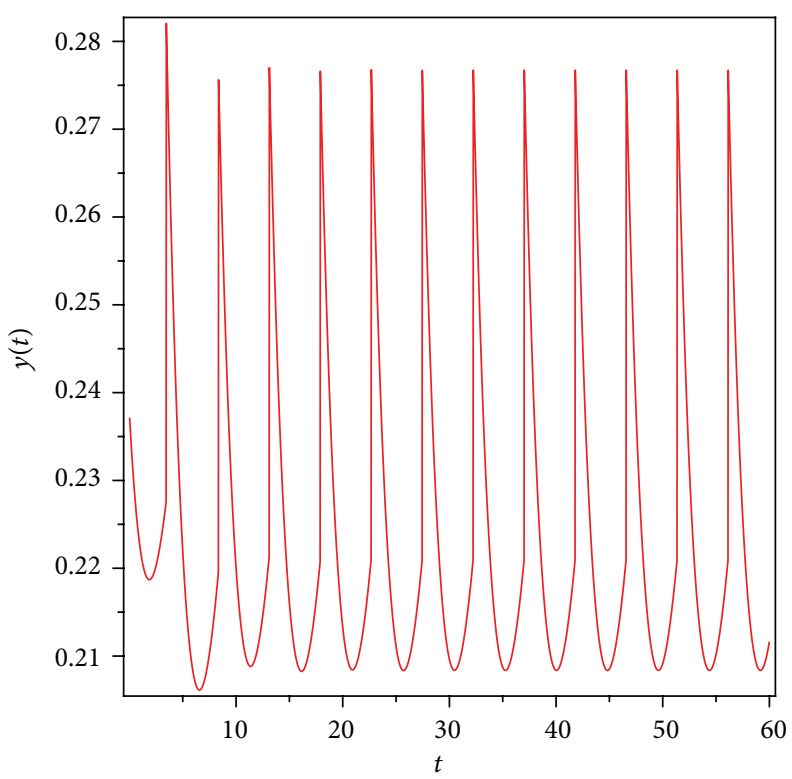

(c) Time series of system (30)

Figure 10: There exists a 1-periodic solution in the area $\Omega_{1}=\left\{(x, y) \mid h_{1}<x<h_{2}\right\}$ corresponding to Figure 5(b).

Case 5. Let $\tau=0.15$, we can easily get the initial point $(0.28,0.2376)$. From Figure 11 corresponding to Figure $5(d)$, clearly, there is no 1-periodic solution in the area $\Omega_{1}=$ $\left\{(x, y) \mid h_{1}<x<h_{2}\right\}$.

Case 6. Let $\tau=0.15$, and the initial point is $(0.28,0.45)$. Obviously, we can find a 1-periodic solution from Figure 12 which corresponds to Figure 6.

All the simulations above show agreement with the results in Section 4.

\section{Conclusion}

This paper establishes a class of integrated pest management model based on state impulse control. In the initial stage of the occurrence of crop pests, that is, the pest density satisfies $x(t) \leq h_{1}$, we use environment protection measures to control pests, such as releasing natural enemies. Once the pest density reaches a higher level $x(t)=h_{2}$, we will adapt a combination of spraying insecticide and releasing natural enemies to control pests. With a short time to finish spraying insecticide and releasing natural enemies which bring out a 


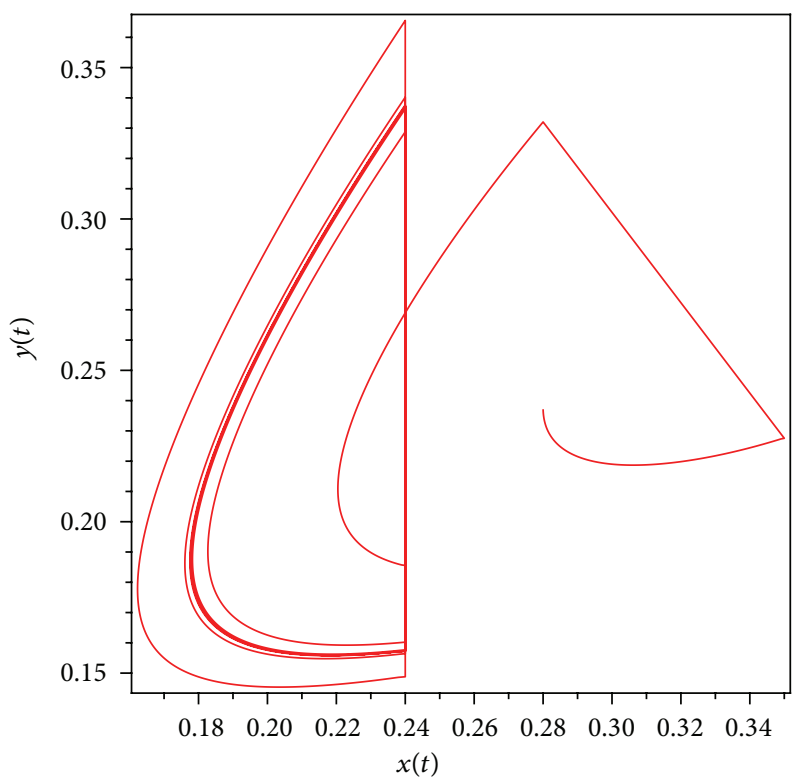

(a) Phase diagram of system (30)

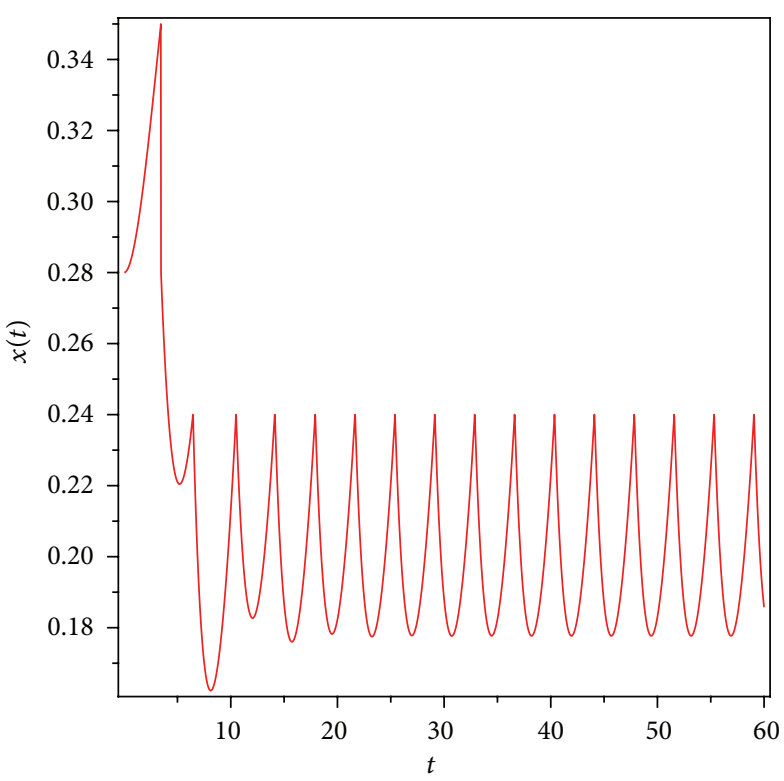

(b) Time series of system (30)

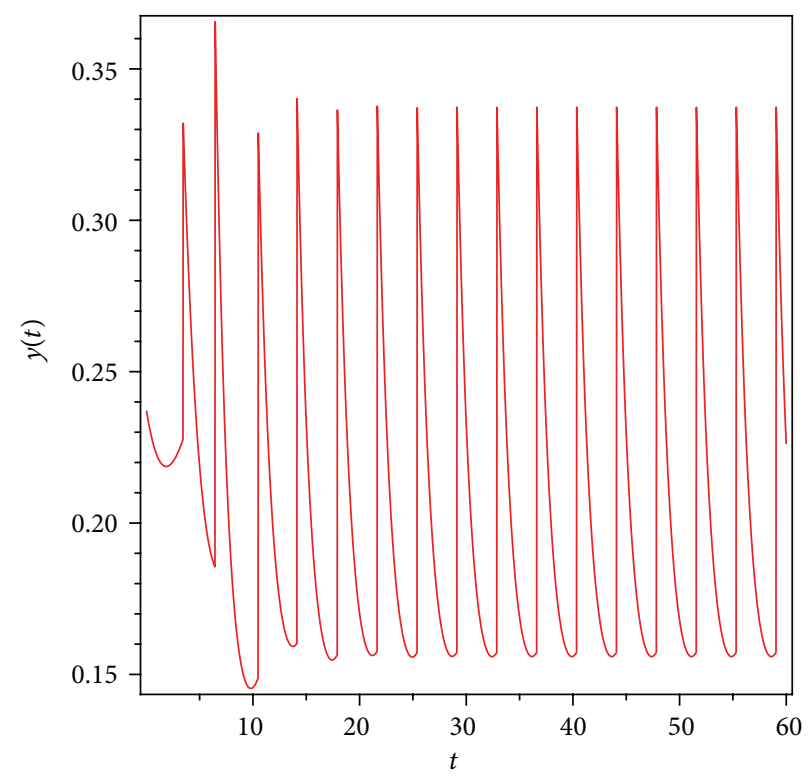

(c) Time series of system (30)

FIGURE 11: 1-periodic solution in the area $\Omega_{1}=\left\{(x, y) \mid h_{1}<x<h_{2}\right\}$ corresponding to Figure 5(d) does not exist.

sharp change in the number of pests and natural enemies, the state impulsive differential system (6) is obtained. Firstly, let the control parameters $p, q, \lambda, \tau$ be zero, we get HollingTanner ecosystem without impulsive effects. By constructing Dulac function, we discussed the stability of the positive equilibrium point $E\left(x^{*}, y^{*}\right)$, and the globally asymptotically stable conditions are given for focal points and nodal point, respectively. If the control parameters $p, q, \lambda, \tau$ are larger than zero, the system (6) is semicontinuous pulse dynamic system. The existence, uniqueness, and stability of the periodic solutions are the research difficulties, and we need to consider all the pulse conditions (the value of $h_{1}, h_{2}$ ) and pulse function and its corresponding qualitative properties of the continuous dynamic system. By introducing the successor function, using impulsive differential geometry theory, we have discussed the existence of periodic solutions of the system (6) with a focus. According to the theory of impulsive differential multiplier Analogue of Poincare Criterion, the conditions of periodic solution with orbit asymptotically stable are given. Since (6) is a two-dimensional dynamical 


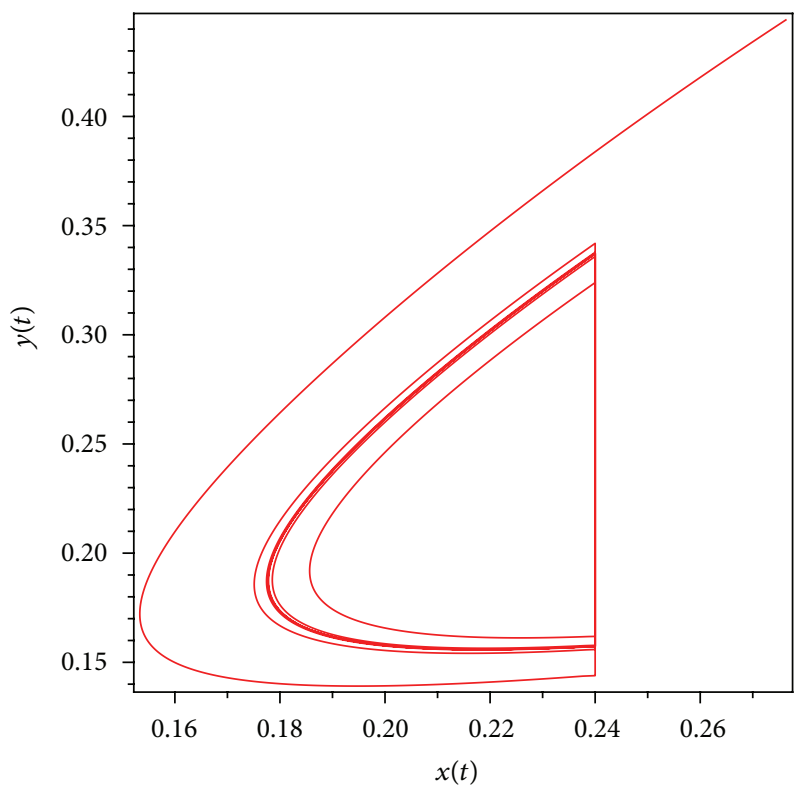

(a) Phase diagram of system (30)

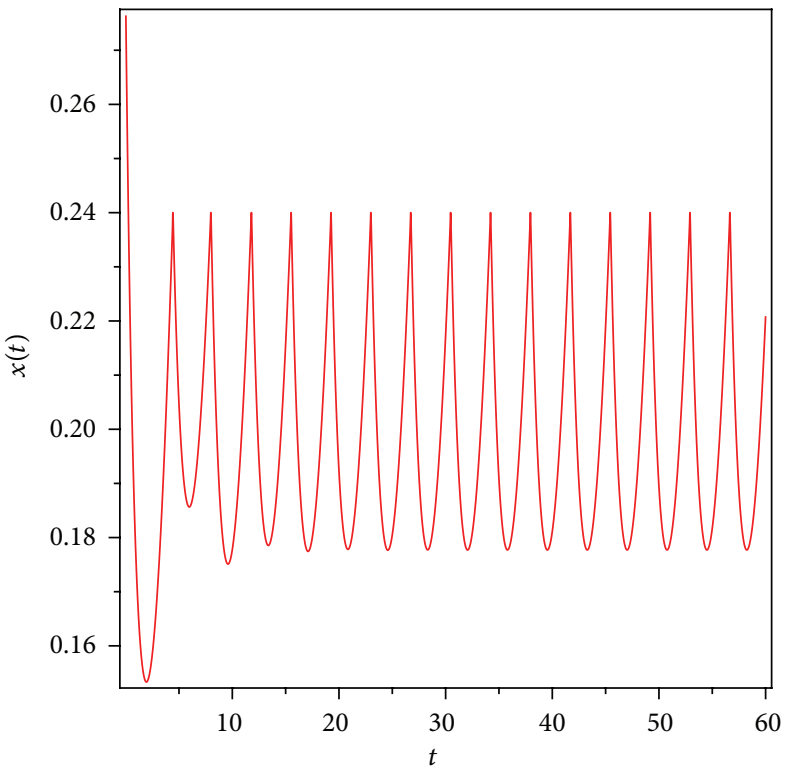

(b) Time series of system (30)

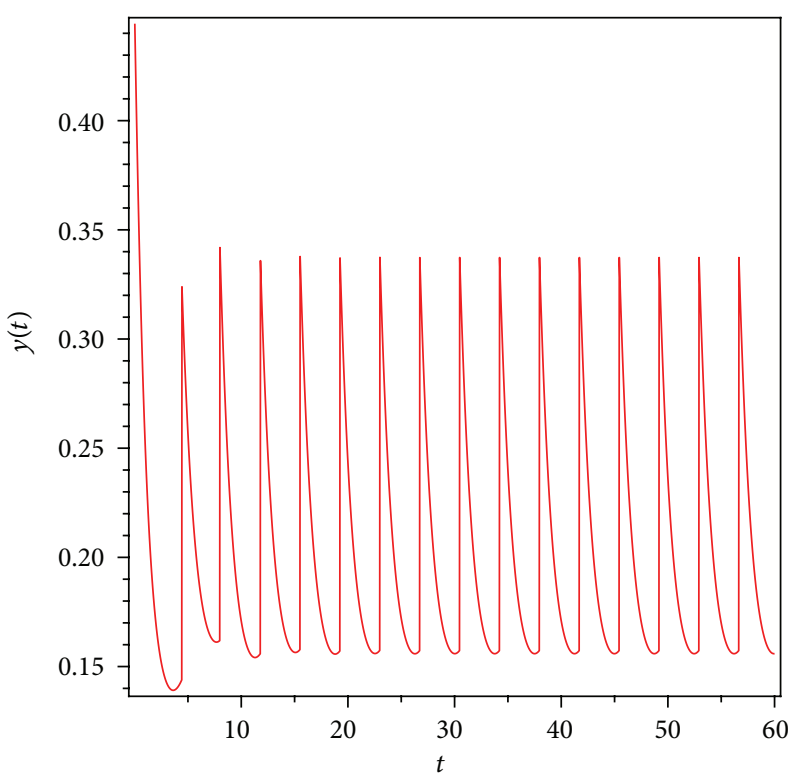

(c) Time series of system (30)

FIGURE 12: The periodic solution corresponding to Figure 6.

system, geometric method is intuitive and effective. How to study high-dimensional ecological dynamic systems by the geometric theory needs to be resolved in the future.

\section{Conflict of Interests}

The authors declare that there is no conflict of interests regarding the publication of this paper.

\section{Acknowledgments}

Wencai Zhao, Tongqian Zhang, and Xinzhu Meng are financially supported by the National Natural Science Foundation of China (no. 11371230), the Shandong Provincial Natural Science Foundation, China (no. ZR2012AM012), and a Project of Shandong Province Higher Educational Science and Technology Program of China (no. J13LI05). Xinzhu Meng is financially supported by the SDUST Research Fund (no. 2011KYTD105).

\section{References}

[1] Agricultural Information Network of China, http://www.agri .gov.c/.

[2] C. C. Yang, C. S. Wang, Y. N. Zheng et al., "Sustained effects of Trichogramma dendrolimi on Ostrinia furnacali," 
Journal of Maize Sciences, vol. 19, no. 1, pp. 139-142, 2011 (Chinese).

[3] D. D. Bainov and P. S. Simeonov, Impulsive Differential Equations: Periodic Solutions and Applications, CRC Press, Boca Raton, Fla, USA, 1993.

[4] V. Lakshmikantham, D. D. Bănov, and P. S. Simeonov, Theory of Impulsive Differential Equations, vol. 6 of Series in Modern Applied Mathematics, World Scientific, Singapore, 1989.

[5] P. S. Simeonov and D. D. Bainov, "Orbital stability of periodic solutions of autonomous systems with impulse effect," International Journal of Systems Science, vol. 19, no. 12, pp. 2561-2585, 1988.

[6] E. M. Bonotto, "LaSalles theorems in impulsive semidynamical systems," Cadernos de Matematica, vol. 9, pp. 157-168, 2008.

[7] E. M. Bonotto and M. Federson, "Topological conjugation and asymptotic stability in impulsive semidynamical systems," Journal of Mathematical Analysis and Applications, vol. 326, no. 2, pp. 869-881, 2007.

[8] J. J. Nieto and D. O'Regan, "Variational approach to impulsive differential equations," Nonlinear Analysis: Real World Applications, vol. 10, no. 2, pp. 680-690, 2009.

[9] J. J. Nieto, "Periodic boundary value problems for first-order impulsive ordinary differential equations," Nonlinear Analysis: Theory, Methods \& Applications, vol. 51, no. 7, pp. 1223-1232, 2002.

[10] L. S. Chen, "Pest control and geometric theory of semicontinuous dynamical system," Journal of Beihua University (Natural Science), vol. 12, no. 1, pp. 1-9, 2011 (Chinese).

[11] L. S. Chen, "Theory and application of semi-continuous dynamical system," Journal of Yulin Normal University (Natural Science), vol. 34, no. 2, pp. 1-9, 2013 (Chinese).

[12] X. Song, M. Hao, and X. Meng, "A stage-structured predatorprey model with disturbing pulse and time delays," Applied Mathematical Modelling, vol. 33, no. 1, pp. 211-223, 2009.

[13] S. Sun and L. Chen, "Mathematical modelling to control a pest population by infected pests," Applied Mathematical Modelling, vol. 33, no. 6, pp. 2864-2873, 2009.

[14] S. Tang and R. A. Cheke, "Models for integrated pest control and their biological implications," Mathematical Biosciences, vol. 215, no. 1, pp. 115-125, 2008.

[15] S. Tang, Y. Xiao, and R. A. Cheke, "Multiple attractors of hostparasitoid models with integrated pest management strategies: eradication, persistence and outbreak," Theoretical Population Biology, vol. 73, no. 2, pp. 181-197, 2008.

[16] B. Liu, Y. Zhang, and L. Chen, "The dynamical behaviors of a Lotka-Volterra predator-prey model concerning integrated pest management," Nonlinear Analysis: Real World Applications, vol. 6, no. 2, pp. 227-243, 2005.

[17] L. Mailleret and F. Grognard, "Global stability and optimisation of a general impulsive biological control model," Mathematical Biosciences, vol. 221, no. 2, pp. 91-100, 2009.

[18] R. Shi and L. Chen, "The study of a ratio-dependent predatorprey model with stage structure in the prey," Nonlinear Dynamics, vol. 58, no. 1-2, pp. 443-451, 2009.

[19] X. Meng, J. Jiao, and L. Chen, "The dynamics of an age structured predator-prey model with disturbing pulse and time delays," Nonlinear Analysis: Real World Applications, vol. 9, no. 2, pp. 547-561, 2008.

[20] X. Meng, Z. Li, and J. J. Nieto, "Dynamic analysis of MichaelisMenten chemostat-type competition models with time delay and pulse in a polluted environment," Journal of Mathematical Chemistry, vol. 47, no. 1, pp. 123-144, 2010.
[21] J. Jiao, W. Long, and L. Chen, "A single stage-structured population model with mature individuals in a polluted environment and pulse input of environmental toxin," Nonlinear Analysis: Real World Applications, vol. 10, no. 5, pp. 3073-3081, 2009.

[22] T. Zhang, X. Meng, and Y. Song, "The dynamics of a highdimensional delayed pest management model with impulsive pesticide input and harvesting prey at different fixed moments," Nonlinear Dynamics, vol. 64, no. 1-2, pp. 1-12, 2011.

[23] T. Q. Zhang, X. Z. Meng, Y. Song, and T. H. Zhang, "A stagestructured predator-prey SI model with disease in the prey and impulsive effects," Mathematical Modelling and Analysis, vol. 18, no. 4, pp. 505-528, 2013.

[24] H. Zhang, J. Jiao, and L. Chen, "Pest management through continuous and impulsive control strategies," BioSystems, vol. 90, no. 2, pp. 350-361, 2007.

[25] H. Zhang, L. Chen, and J. J. Nieto, "A delayed epidemic model with stage-structure and pulses for pest management strategy," Nonlinear Analysis: Real World Applications, vol. 9, no. 4, pp. 1714-1726, 2008.

[26] J.-J. Jiao, L.-S. Chen, J. J. Nieto, and A. Torres, "Permanence and global attractivity of stage-structured predator-prey model with continuous harvesting on predator and impulsive stocking on prey," Applied Mathematics and Mechanics, vol. 29, no. 5, pp. 653-663, 2008.

[27] S. Tang, Y. Xiao, L. Chen, and R. A. Cheke, "Integrated pest management models and their dynamical behaviour," Bulletin of Mathematical Biology, vol. 67, no. 1, pp. 115-135, 2005.

[28] S. Tang and R. A. Cheke, "State-dependent impulsive models of integrated pest management (IPM) strategies and their dynamic consequences," Journal of Mathematical Biology, vol. 50, no. 3, pp. 257-292, 2005.

[29] G. Zeng, L. Chen, and L. Sun, "Existence of periodic solution of order one of planar impulsive autonomous system," Journal of Computational and Applied Mathematics, vol. 186, no. 2, pp. 466-481, 2006.

[30] L. Zhao, L. Chen, and Q. Zhang, “The geometrical analysis of a predator-prey model with two state impulses," Mathematical Biosciences, vol. 238, no. 2, pp. 55-64, 2012.

[31] L. Nie, Z. Teng, L. Hu, and J. Peng, "Existence and stability of periodic solution of a predator-prey model with statedependent impulsive effects," Mathematics and Computers in Simulation, vol. 79, no. 7, pp. 2122-2134, 2009.

[32] L. Nie, Z. Teng, L. Hu, and J. Peng, "Qualitative analysis of a modified Leslie-Gower and Holling-type II predatorprey model with state dependent impulsive effects," Nonlinear Analysis: Real World Applications, vol. 11, no. 3, pp. 1364-1373, 2010.

[33] B. Liu, Y. Tian, and B. Kang, "Dynamics on a Holling II predator-prey model with state-dependent impulsive control," International Journal of Biomathematics, vol. 5, no. 3, Article ID 1260006, 2012.

[34] C. Dai, M. Zhao, and L. Chen, "Homoclinic bifurcation in semi-continuous dynamic systems," International Journal of Biomathematics, vol. 5, no. 6, Article ID 1250059, 2012.

[35] J. B. Fu and Y. Z. Wang, "The mathematical study of pest management strategy," Discrete Dynamics in Nature and Society, vol. 2012, Article ID 251942, 19 pages, 2012.

[36] C. Wei and L. Chen, "Periodic solution of prey-predator model with Beddington-DeAngelis functional response and impulsive state feedback control," Journal of Applied Mathematics, vol. 2012, Article ID 607105, 17 pages, 2012. 
[37] C. Wei and L. Chen, "Heteroclinic bifurcations of a preypredator fishery model with impulsive harvesting," International Journal of Biomathematics, vol. 6, no. 5, Article ID 1350031, 2013.

[38] L. S. Chen, X. Z. Meng, and J. J. Jiao, Biological Dynamics, Science Press, Beijing, China, 2009.

[39] S. B. Hsu and T. W. Huang, "Global stability for a class of predator-prey systems," SIAM Journal on Applied Mathematics, vol. 55, no. 3, pp. 763-783, 1995. 


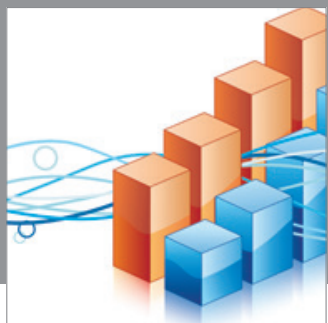

Advances in

Operations Research

mansans

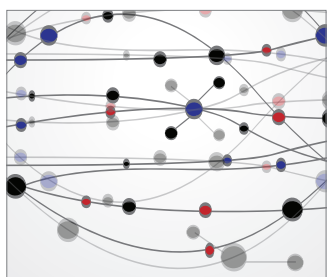

The Scientific World Journal
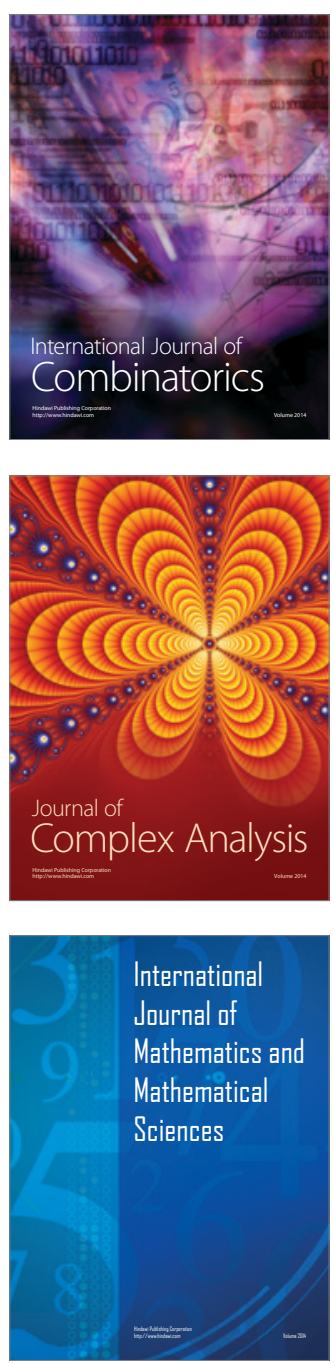
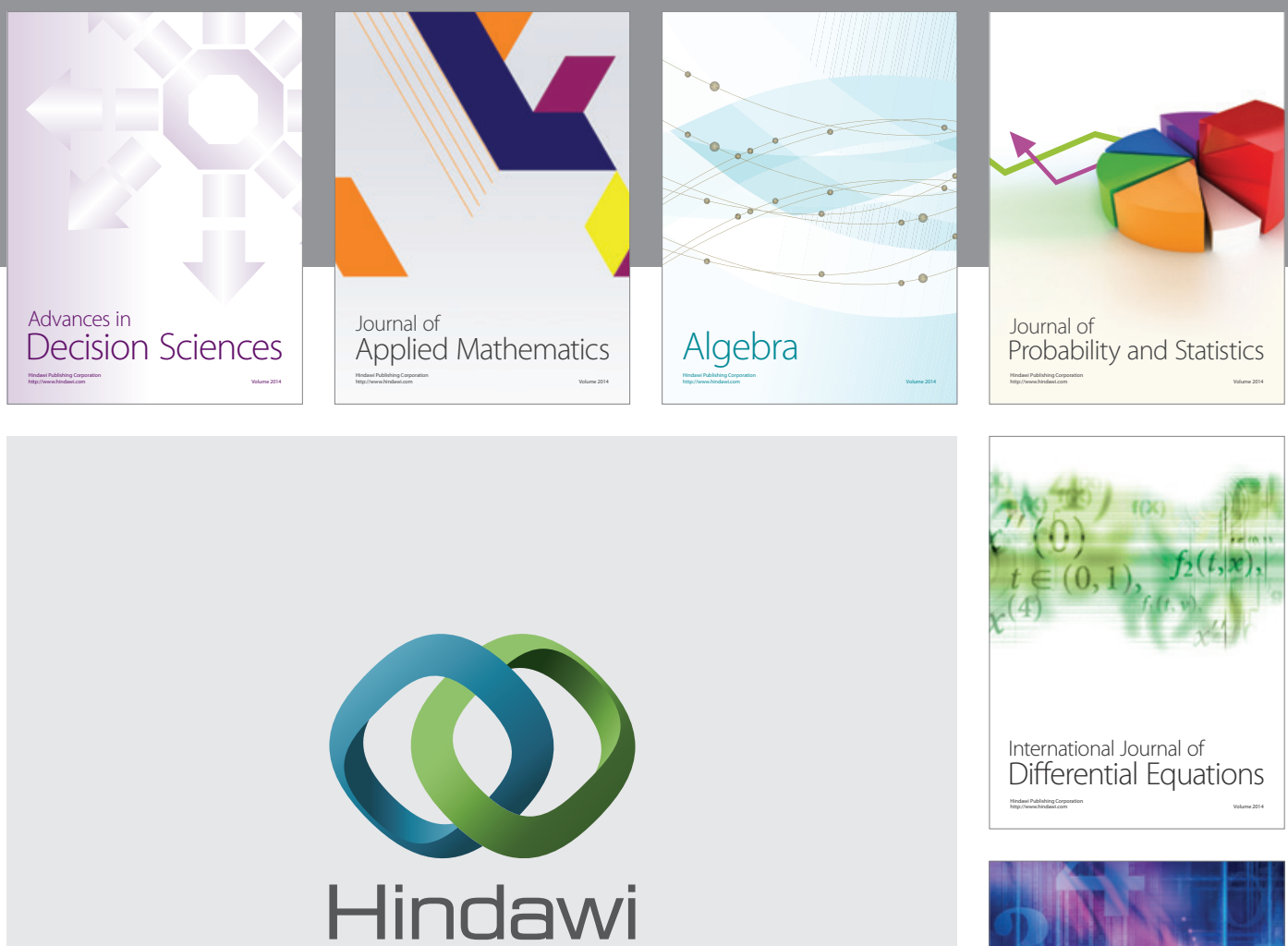

Submit your manuscripts at http://www.hindawi.com
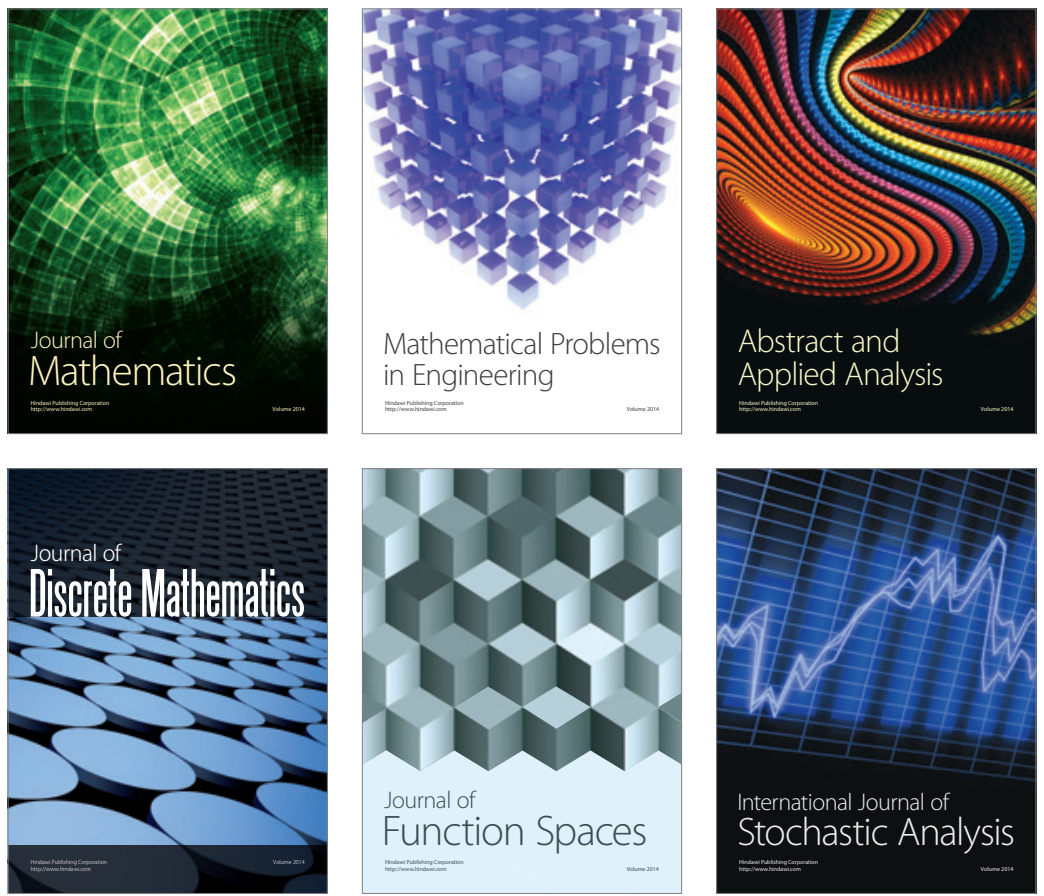

Journal of

Function Spaces

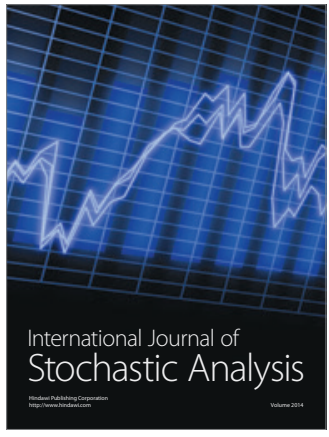

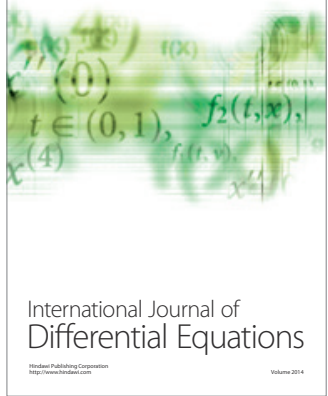
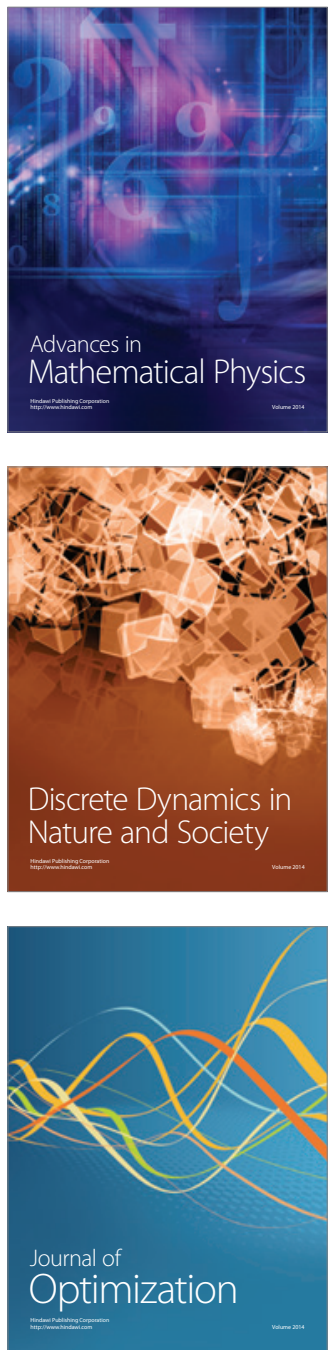